\title{
Energy Aware Trajectory Optimization for Aerial Base Stations
}

\author{
Xiaoye Jing, Student Member, IEEE, Jingcong Sun, Christos Masouros, Senior Member, IEEE
}

\begin{abstract}
By fully exploiting the mobility of unmanned aerial vehicles (UAVs), UAV-based aerial base stations (BSs) can move closer to ground users to achieve better communication conditions. In this paper, we consider a scenario where an aerial BS is dispatched for satisfying the data request of a maximum number of ground users, weighted according to their data demand, before exhausting its on-board energy resources. The resulting trajectory optimization problem is a mixed integer non-linear problem (MINLP) which is challenging solve. Specifically, there are coupling constraints which cannot be solved directly. We exploit a penalty decomposition method to reformulate the optimization formulation into a new form and use block coordinate descent technique to decompose the problem into sub-problems. Then, successive convex approximation technique is applied to tackle non-convex constraints. Finally, we propose a double-loop iterative algorithm for the UAV trajectory design. In addition, to achieve a better coverage performance, the problem of designing the initial trajectory for the UAV trajectory is considered. In the results section, UAV trajectories with the proposed algorithm are shown. Numerical results show the coverage performance with the proposed schemes compared to the benchmarks.
\end{abstract}

Index Terms-Unmanned aerial vehicles, aerial base stations, trajectory optimization, user scheduling and association

\section{INTRODUCTION}

$\mathbf{I}$ $\mathrm{N}$ particular, unmanned aerial vehicles (UAVs) serving as aerial base stations (BSs) have gradually been an important part of wireless service providers, thanks to their flexibility, interoperability and the favourable line-of-sight (LoS) communication conditions [1]-[4]. Aerial BSs can be deployed to ease the burden of existing cellular systems in extremely crowded areas, and applied in emergency or disaster scenarios where terrestrial communication infrastructures are damaged or even totally destroyed [2], [5]. Deploying UAV as a hub or an aerial BS provides communication connectivity between users and the core network. The use of UAV in this area includes two typical cases. The more common case is that the UAV is considered as an aerial BS which connects with users and delivers traffic to or from the core network. The second case is deploying the UAV as a fronthaul or backhaul hub which is the relay between users and the core network elements.

Some researches focus on static aerial BSs. The authors in [6] and [7] studied the relationship between the altitude of a

This work was supported by the European Union's Horizon 2020 research and innovation programme under the Marie Skłodowska-Curie grant agreement No 812991.

X. Jing and C. Masouros are with the Department of Electronics and Electrical Engineering, University College London, London WC1E 7JE, U.K. (e-mail: x.jing@ucl.ac.uk; chris.masouros@ieee.org).

Jingcong Sun is from Huawei, Shenzheng, China (emial:jingcong.sun.16@alumni.ucl.ac.uk). static UAV and its corresponding coverage area. The work in [8] then extended the number of deployed UAVs to two by considering the effect of inter-cell interference (ICI). In [9][13], various algorithms have been proposed to maximize the number of users that can be covered by static UAVs.

In order to fully exploit the potential of aerial BSs, recent research focus has shifted to the high mobility of aerial BSs. With the exploitation of controllable UAV mobility, distances between the aerial BS and users can be reduced and then communication path loss can be greatly descreased [14]. It is worth mentioning that the endurance of aerial BSs is fundamentally constrained by the limited built-in battery energy, and the efficient use of on-board energy is thus of paramount importance in UAV related applications [2], [15]. Without considering the propulsion energy for supporting the movement of UAVs, efficient usage of energy for communication related functions have been studied in [16]-[18]. Authors in [19], [20] took into account the propulsion power consumption. On one hand, compared to static aerial BSs, moving aerial BSs can fly close to ground users to improve the transmission channel quality, thus serving more users. On the other hand, the trajectory of UAV is intrinsically constrained by its limited on-board energy which becomes an obstruction for serving more users.

In this paper, we consider a scenario that an aerial BS is dispatched from a charging base to meet the service requirement of ground users. After flying follows a proposed trajectory, the UAV is required to finish at the charging base for recharging before exhausting its on-board energy. We assume that a user is satisfied only when its entire data requested is delivered. Fixed-wing UAVs which have a higher speed than rotary-wing UAVs are chosen as the carrier for aerial BSs in this paper [2]. Our aim is satisfying the data demand of a maximum number of ground users, weighted according to their data demand, with a limited on-board energy resource via optimizing the UAV flying trajectory. The trajectory optimization includes the joint optimization of the user communication scheduling and association, UAV flying status related parameters, UAV completion time, as well as communication time among users, and UAV transmit power ${ }^{1}$. Different from the existing research in fixed-wing UAV trajectory design, time is variable and an optimization parameter in our problem. This is essential for problems where energy is studied. Since the trajectories

\footnotetext{
${ }^{1}$ Note that part of this work has been part of the thesis in [21]. Compared to [21], our work extends the signalling and optimization to include variable path slot durations, and further proposes a tailored power allocation. Accordingly, we propose a novel algorithm to address the optimization problem compared to the thesis. Additionally, we address the issue of initial trajectory design, where we design two initial trajectories in this work.
} 
are not constrained by pre-determined completion time, the coverage performance study influenced by energy will be more meaningful. For instance, in previous study where time is fixed, if the completion time is small, the UAV is not able to consume all energy at the end of the service, which constrains the UAV coverage ability. On the other hand, with increased completion time, the battery capacity may not be enough to support the whole service.

For clarity, the main contributions of this paper are summarized as follows

- First, we formulate the trajectory optimization problem. We apply a path discretization method to decompose the UAV overall path into sub paths and define optimization variables according to these sub paths [22]-[24]. Therefore, the optimization problem can be addressed via optimizing variables, i.e. the variables about the user communication scheduling and association, the completion time, and the communication time among users, the UAV location, the UAV velocity, the UAV acceleration, the transmit power in every sub path.

- Due to the fact that the completion time and communication time among users are optimization variables in our optimization problem, the constraints of the problem formulation contains two types of couplings. Firstly, there are equality coupling constraints between a) time and velocity, b) time and acceleration. By exploiting a penalty decomposition method [25], [26], we introduce new variables to replace these couplings and add these coupling constraints as penalty factors into the objective function. An iterative algorithm is proposed for applying this penalty method. Secondly, some inequality constraints include couplings between a) time and transmit power, b) time, data rate, and user communication scheduling and association. We apply an iterative algorithm based on block coordinate descent (BCD) technique to tackle these couplings. Specifically, the entire optimization variables will be divided into four subsets with four sub-problems and the four subsets are alternately optimized within each iteration. However, it is still challenging to address some sub-problems due to some non-convex constraints and objectives. Successive convex approximation (SCA) technique [27] is then utilized in these non-convex constraints and objectives, and then we transform them into convex forms. Finally, a double-loop iterative algorithm is proposed for the entire optimization problem based on the two iterative algorithms above.

- Furthermore, since the proposed algorithm is a doubleloop iterative algorithm, we need an initial trajectory to start the iterative process. A feasible circular initial trajectory (CIT) which is simple to implement is firstly proposed. Furthermore, to improve the coverage performance, we design another initial trajectory, which can give all users a relatively fair chance to be scheduled and satisfy all UAV flying status constraints, as well as the energy constraint. Simulation results demonstrating the comparison between these two initial trajectories are shown in results section.

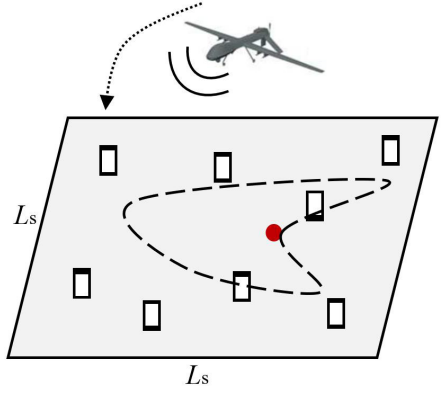

Fig. 1. Aerial BS serving ground users.

We add a list of the important insights obtained from our proposed algorithm and the simulation results as follows

- Comparing our proposed trajectory with the UAV trajectory based on a pre-specified completion time, we can see that, allowing variable time between path slots, relaxes the optimization and improves the coverage probability of the proposed algorithm respect to the conventional UAV trajectory.

- The gains of our energy-constrained trajectory design are tightly related to the on-board battery capacity. An increase in the available energy results in a larger trajectory and completion time, which enables satisfying the quality of service requirements of more users.

- Our proposed penalty method introduces a scalable performance-complexity trade-off. Our results show that a bigger absolute value of the penalty factors results in a higher coverage probability, at the expense of a higher number of optimization iterations. By tuning these factors one can obtain a favourable trade-off between coverage performance and algorithm processing time.

The remainder of this paper is organized as follows. Section

II introduces system model and formulates the problem. The proposed iterative algorithm for the optimization problem is shown in Section III. Section IV introduces a modified circular initial trajectory based on the path discretization and a designed initial trajectory for achieving better serving performance. In Section V, benefits of the proposed techniques are evaluated with numerical results. Finally, the paper is concluded in Section VI.

\section{SYSTEM MODEL AND PROBLEM FORMULATION}

\section{A. System Model}

As shown in Fig. 1, we consider a square geographical target area of a dimension $L_{s}$ by $L_{s}$, containing $M$ ground users. We assume that users have low-mobility and are uniformly distributed within the target area. We consider a 3-D Cartesian coordinate system where the horizontal location of the $m$ th user is $\mathbf{w}_{m}=\left[x_{m}, y_{m}\right] \in \mathbb{R}^{1 \times 2}$. The UAV is able to charge its battery at the charging base, represented by the red dot as shown in Fig. 1. In this paper, we assume the center of the target area as the charging base station, where is $\mathbf{s}_{0}=\left[\frac{L_{\mathrm{s}}}{2}, \frac{L_{\mathrm{s}}}{2}\right]$. Following the proposed trajectory, the aerial BS tries to cover as many users as possible before exhausting its on-board energy and its flying finishes at the base for 
recharging ${ }^{2}$.

Since a trajectory is characterized by the geographical positions together with the flying velocity and acceleration corresponding to the path, we describe the UAV trajectory by employing the path discretization method in [22]-[24] by applying the discrete linear state-space approximation to the UAV trajectory. With path discretization, the UAV continuoustime path is discretized into $N$ sub paths. Each sub path is a line segment, presented by $N+1$ locations, where $\mathbf{s}[n]=\left[s_{x}[n], s_{y}[n]\right] \in \mathbb{R}^{1 \times 2}, n=1,2, \ldots, N+1$, with $\mathbf{s}[1]=\mathbf{s}_{0}$ and $\mathbf{s}[N+1]=\mathbf{s}_{0}$. The length of each line segment should be chosen with a constraint $\|\mathbf{s}[n+1]-\mathbf{s}[n]\| \leq \Delta$, where $\Delta$ is chosen as $\Delta \ll H$ [22], [23]. We assume that the UAV flies with a fixed altitude $H$, where $H$ could correspond to the minimum altitude required for safe operation. Therefore, within each sub path, the distance between the UAV and each user is approximately unchanged. To obtain a more tractable form with a finite number of optimization variables, we denote optimization variables corresponding to these sub paths. The UAV flying time in $n$-th path slot is $T[n]$. The UAV velocity at location $\mathbf{s}[n]$ is $\mathbf{v}[n]$ and the UAV acceleration in $n$-th path slot is $\mathbf{a}[n]$. The relationship among $\mathbf{s}[n], \mathbf{v}[n]$ and $\mathbf{a}[n]$ can be described by the following two equations

$$
\begin{gathered}
\mathbf{s}[n+1]-\mathbf{s}[n]=\mathbf{v}[n] T[n]+\frac{1}{2} \mathbf{a}[n] T^{2}[n], \forall n, \\
\mathbf{v}[n+1]-\mathbf{v}[n]=\mathbf{a}[n] T[n], \forall n .
\end{gathered}
$$

For simplicity, we assume that the air-to-ground (AtG) links are dominated by LoS channels [28]-[30], but is also one of the main reason that motivates us to deploy flying BS. The distance between the UAV and $m$-th user in $n$-th path slot is given by

$$
d_{m}[n]=\sqrt{H^{2}+\left\|\mathbf{s}[n]-\mathbf{w}_{m}\right\|^{2}} .
$$

Correspondingly, the path-varying channel for $m$-th user in $n$-th path slot at time $t$ is expressed as

$$
h_{m}[n, t]=\frac{\beta_{0}\left|g_{m}[n, t]\right|^{2}}{d_{m}[n]^{2}}=\frac{\beta_{0}\left|g_{m}[n, t]\right|^{2}}{H^{2}+\left\|\mathbf{s}[n]-\mathbf{w}_{m}\right\|^{2}},
$$

where we denote $\beta_{0}$ as the channel power at the reference distance $d_{0}=1 \mathrm{~m}, g_{m}[n, t]$ as the channel coefficient which captures the shadowing and the small-scale fading effect in $n$-th path slot at time $t$ with $\mathbb{E}\left[\left|g_{m}[n, t]\right|^{2}\right]=1$ [22]. The achievable transmission rate in bits per second between $m$-th user and the UAV in $n$-th path slot at time $t$ is then expressed as

$$
r_{m}[n]=B \log _{2}\left(1+\frac{P_{\mathrm{t}}[n] h_{m}[n, t]}{\sigma^{2}}\right),
$$

where $B, P_{\mathrm{t}}[n]$ and $\sigma^{2}$ denote the total available bandwidth, the transmit power of the UAV and the noise power respectively.

We define a binary variable $\alpha_{m}[n]$ indicating the communication scheduling and association status of $m$-th user in $n$-th

\footnotetext{
${ }^{2}$ Note that part of this work has been submitted in [24]. Different from [24], our trajectory optimization work bases on fixed-wing UAV. Thus, the formulation in this work contains coupling equality constraints and we propose a double-loop algorithm for these couplings.
}

path slot. Specifically, if $\alpha_{m}[n]=1$, the $m$-th user is served by the UAV in $n$-th path slot, and otherwise $\alpha_{m}[n]=0$. We assume that at most one of the $M$ users is associated with the aerial BS in each path slot, which can be expressed as

$$
\sum_{m=1}^{M} \alpha_{m}[n] \leq 1, \forall n
$$

The aggregated communication throughput of $m$-th user in $n$-th path slot is expressed as

$$
\tilde{R}_{m}[n]=\int_{0}^{T[n]} \alpha_{m}[n] B \log _{2}\left(1+\frac{P_{\mathrm{t}}[n] h_{m}[n, t]}{\sigma^{2}}\right) d t .
$$

Since $h_{m}[n, t]$ is a variable in $n$-th path slot at different time, we obtain the expected throughput to replace $\tilde{R}_{m}[n]$ as

$$
\begin{aligned}
& \bar{R}_{m}[n] \\
& =\int_{0}^{T[n]} \alpha_{m}[n] B \mathbb{E}\left[\log _{2}\left(1+\frac{P_{\mathrm{t}}[n] h_{m}[n, t]}{\sigma^{2}}\right)\right] d t \\
& \approx \int_{0}^{T[n]} \alpha_{m}[n] B \log _{2}\left(1+\frac{P_{\mathrm{t}}[n] \mathbb{E}\left[h_{m}[n, t]\right]}{\sigma^{2}}\right) d t \\
& =\int_{0}^{T[n]} \alpha_{m}[n] B \log _{2}\left(1+\frac{P_{\mathrm{t}}[n] \zeta_{0}}{H^{2}+\left\|\mathbf{s}[n]-\mathbf{w}_{m}\right\|^{2}}\right) d t,
\end{aligned}
$$

where $\zeta_{0}=\frac{\beta_{0}}{\sigma^{2}}$ denotes the referenced received signal-to-noise (SNR). We note that the above throughput expression requires the knowledge of the expectation value of channel's small scale fading. The communication throughput of $m$-th user in $n$-th path slot is

$$
R_{m}[n]=\alpha_{m}[n] T[n] B \log _{2}\left(1+\frac{P_{\mathrm{t}}[n] \zeta_{0}}{H^{2}+\left\|\mathbf{s}[n]-\mathbf{w}_{m}\right\|^{2}}\right) .
$$

Finally, the achievable total data received of $m$-th user in the unit of bits is thus given by

$$
R_{m}=\sum_{n=1}^{N} \alpha_{m}[n] T[n] B \log _{2}\left(1+\frac{P_{\mathrm{t}}[n] \zeta_{0}}{H^{2}+\left\|\mathbf{s}[n]-\mathbf{w}_{m}\right\|^{2}}\right) .
$$

The power consumption of the UAV consists of two parts, i.e., the power consumed for communication and for supporting the UAV mobility. The communication-related power in $n$-th path slot is denoted by $P_{\mathrm{t}}[n]$. Propulsion power consumption depends on the UAV flying status, and a theoretical model was derived in [19]. We adopt the energy model in this paper and then the propulsion power model in $n$-th path slot is

$$
P_{\mathrm{p}}[n]=c_{1}\|\mathbf{v}[n]\|^{3}+\frac{c_{2}}{\|\mathbf{v}[n]\|}\left(1+\frac{\|\mathbf{a}[n]\|^{2}}{G^{2}}\right),
$$

where $c_{1}$ and $c_{2}$ are constants related to UAV's design, air density, etc., and $G=9.8 \mathrm{~m} / \mathrm{s}^{2}$ represents the gravitational acceleration. Correspondingly, the total consumed energy is

$$
E_{\mathrm{c}}=\sum_{n=1}^{N} P_{\mathrm{p}}[n] T[n]+\sum_{n=1}^{N} P_{\mathrm{t}}[n] T[n] .
$$




\section{B. Problem Formulation}

Our goal is to maximize the number of data-satisfied users with limited energy by the joint optimization of 1) the user communication scheduling and association, 2) UAV flying status related parameters, 3) UAV completion time, as well as communication time among users, and 4) UAV transmit power. The problem is firstly formulated as

$$
\begin{aligned}
(\mathrm{P} 1): \quad & \max _{\substack{\left.\alpha_{m}[n], \mathbf{s}[n], \mathbf{v}[n], \mathbf{a}[n] \\
T[n], P_{\mathrm{t}} n\right], \eta_{m}}}: \sum_{m \in M} \omega_{m} \eta_{m} \\
\text { s.t. } \quad & R_{m} \geq \eta_{m} Q_{m}, \forall m, \\
& \eta_{m} \in\{0,1\}, \forall m, \\
& \alpha_{m}[n] \in\{0,1\}, \forall n, \forall m, \\
& \sum_{m=1}^{M} \alpha_{m}[n] \leq 1, \forall n, \\
& \sum_{n=1}^{N}\left(c_{1}\|\mathbf{v}[n]\|^{3}+\frac{c_{2}}{\|\mathbf{v}[n]\|}\left(1+\frac{\|\mathbf{a}[n]\|^{2}}{G^{2}}\right)\right. \\
& +\sum_{n=1}^{N} P_{\mathrm{t}}[n] T[n] \leq E_{\mathrm{tot}}, \\
& \mathbf{s}[1]=\mathbf{s}[N+13 \mathrm{a}] \\
& \mathbf{s}[n+1]-\mathbf{s}[n] \\
& =\mathbf{v}[n] T[n]+\frac{1}{2} \mathbf{a}[n] T^{2}[n], \forall n, \\
& \mathbf{v}[n+1]-\mathbf{v}[n]=\mathbf{a}[n] T[n], \forall n, \\
& \mathbf{v}[N+1]=\mathbf{v}_{0}, \\
& \|\mathbf{v}[n]\| \leq v_{\max }, \forall n, \\
& \|\mathbf{v}[n]\| \geq v_{\min }, \forall n, \\
& \|\mathbf{a}[n]\| \leq a_{\max }, \forall n, \\
& \|\mathbf{s}[n+1]-\mathbf{s}[n]\| \leq \Delta, \forall n, \\
& T[n]>0, \forall n, \\
& 0 \leq P_{\mathrm{t}}[n] \leq P_{\max }, \forall n .
\end{aligned}
$$

A binary variable $\eta_{m}$ is utilized for indicating whether the data demand of $m$-th user is satisfied or not. We assume that the data requested by $m$-th user is $Q_{m}, \eta_{m}=1$ when $R_{m} \geq Q_{m}$, and otherwise, $\eta_{m}=0$. $E_{\text {tot }}$ denotes the total on-board energy of the UAV; $\mathbf{v}_{0}, v_{\max }, v_{\min }$ and $a_{\max }$ denote the final velocity, maximum allowed speed, minimum required speed and maximum allowed acceleration of the fixed-wing UAV respectively; $P_{\max }$ is the maximum transmit power. $\omega_{m}$ is a weighted factor of $m$-th user, where $\omega_{m}=\frac{Q_{m}}{Q_{1}+Q_{2}+\ldots Q_{M}}$. By applying the weighted factors, users with larger data demand, which are usually more difficult to be satisfied, also contribute greater to the objective value. Therefore, all the ground users have a fair opportunity to be scheduled and associated. Constraint (13k) and (13n) mean that UAV cannot stay at one location, since the fixed-wing UAV cannot hover in one place. As can be seen in the constraint (13a), when the total received data of $m$-th user is equal or larger than the required data $Q_{m}, \eta_{m}=1$ and the objective value is increased by $\omega_{m}$ correspondingly. However, when the data demand of $m$-th user is not met, $\eta_{m}=0$ and the objective value remains the same. Note that, (13e) guarantees that the
UAV total consumed energy should be no larger than its onboard energy. According to (13f), the UAV is dispatched from the charging base at the first path slot, and should fly back to the base for recharging at the end of the trajectory. In addition, the UAV mobility is governed by the velocity constraints as specified in (13j)-(131).

\section{PROPOSED ITERATIVE ALGORITHM FOR COVERAGE MAXIMIZATION}

In Section II, we propose the system model for our problem and formulate the optimization problem in $\mathrm{P} 1$. In problem $\mathrm{P} 1$, the constraints $(13 \mathrm{~g})$ and $(13 \mathrm{~h})$ are equality constraints which contain couplings between a) $T[n]$ and $\mathbf{v}[n]$ and between b) $T[n]$ and $\mathbf{a}[n]$. Both (13a) and (13e) are inequality constraints which include couplings and are non-convex. Furthermore, constraint (13k) is non-convex. Therefore, problem P1 is nonconvex and it is difficult to find its globally optimal solution. In this section, we propose an algorithm to find a solution for $\mathrm{P} 1$ based on an iterative process.

\section{A. The Penalty Method}

As we mentioned above, there are couplings between variables in P1. A typical method to decouple multiple variables in UAV trajectory optimization is the BCD technique. However, $(13 \mathrm{~g})$ and (13h) are equality coupling constraints. Due to we cannot optimize some variables of an equation via one subproblem and optimize the remaining variables of the same equation via another sub-problem, BCD technique is not available here. Consequently, we exploit an algorithm which is a high quality solution for these equality coupling constraints. Specifically, we introduce auxiliary variables and constraints to replace couplings of the original equations and then decouple these couplings based on a penalty way.

Firstly, we introduce $3 N$ new variables $\mathbf{f}_{1}[n], \mathbf{f}_{2}[n], \mathbf{f}_{3}[n]$ and $3 N$ new equations, then rewrite $\mathrm{P} 1$ as

$$
\begin{aligned}
\left(\mathrm{P}^{\prime}\right): & \max _{\substack{\alpha_{m}[n], \mathbf{s}[n], \mathbf{v}[n], \mathbf{a}[n], T[n] \\
\mathbf{f}_{1}[n], \mathbf{f}_{2}[n], \mathbf{f}_{3}[n], P_{\mathrm{t}}[n], \eta_{m}}}: \sum_{m \in M} \omega_{m} \eta_{m} \\
\text { s.t. } & \mathbf{s}[n+1]-\mathbf{s}[n]=\mathbf{f}_{1}[n]+\mathbf{f}_{2}[n], \forall n, \\
& \mathbf{v}[n+1]-\mathbf{v}[n]=\mathbf{f}_{3}[n], \forall n, \\
& \mathbf{f}_{1}[n]=\mathbf{v}[n] T[n], \forall n, \\
& \mathbf{f}_{2}[n]=\frac{1}{2} \mathbf{a}[n] T^{2}[n], \forall n, \\
& \mathbf{f}_{3}[n]=\mathbf{a}[n] T[n], \forall n, \\
& (13 \mathrm{a})-(13 \mathrm{f}) \text { and }(13 \mathrm{i})-(13 \mathrm{o}) .
\end{aligned}
$$

It can be seen above, the couplings between a) $T[n]$ and $\mathbf{v}[n]$, b) $T[n]$ and $\mathbf{a}[n]$ in (13g) and (13h) are decoupled in (14a) and (14b). However, new couplings are introduced in (14c), (14d) and (14e). Therefore, we introduce $3 N$ penalty parameters $\rho_{1}[n], \rho_{2}[n]$ and $\rho_{3}[n]$, where $\rho_{1}[n], \rho_{2}[n], \rho_{3}[n]$ $>0, n \in N$ and rewrite the objective function of $\mathrm{P} 1^{\prime}$ as (15) shown at the top of the next page. Thus, $\mathrm{P} 1^{\prime}$ is converted to

$$
\begin{aligned}
& \left(\mathrm{P}^{\prime \prime}\right): \max _{\substack{\alpha_{m}[n], \mathbf{s}[n], \mathbf{v}[n], \mathbf{a}[n], T[n] \\
\mathrm{f}_{1}[n], \mathbf{f}_{2}[n], \mathbf{f}_{3}[n], P_{\mathrm{t}}[n], \eta_{m}}}: F \\
& \text { s.t. } \quad(13 a)-(13 f),(13 i)-(13 o),(14 a) \text { and (14b). }
\end{aligned}
$$




$$
F=\omega_{m} \eta_{m}-\sum_{n \in N} \frac{1}{2 \rho_{1}[n]}\left\|\mathbf{f}_{1}[n]-\mathbf{v}[n] T[n]\right\|^{2}-\sum_{n \in N} \frac{1}{2 \rho_{2}[n]}\left\|\mathbf{f}_{2}[n]-\frac{1}{2} \mathbf{a}[n] T^{2}[n]\right\|^{2}-\sum_{n \in N} \frac{1}{2 \rho_{3}[n]}\left\|\mathbf{f}_{3}[n]-\mathbf{a}[n] T[n]\right\|^{2} .
$$

It can be seen that, as $\rho_{1}[n], \rho_{2}[n]$ and $\rho_{3}[n]$ tend to 0 , the convergent solution of $\mathrm{P} 1^{\prime \prime}$ is an optimal solution of the original problem. However, when $\rho_{1}[n], \rho_{2}[n]$ and $\rho_{3}[n]$ are very small, the process may cause difficulties in computation [25], [26]. Therefore, we introduce $3 N$ multiplier variables $\boldsymbol{\lambda}_{1}[n], \boldsymbol{\lambda}_{2}[n]$ and $\boldsymbol{\lambda}_{3}[n] \in \mathbb{R}^{1 \times 2}, n \in N$ and rewrite an objective function as (16) shown at the top of the next page for $\mathrm{P} 1^{\prime \prime}$ by recovering an exact optimal solution of problem $\mathrm{P} 1^{\prime \prime}$ and define the following augmented Lagrange penalty problem

$$
\begin{aligned}
& \left(\mathrm{P} 1^{\prime \prime \prime}\right): \quad \max _{\substack{\alpha_{m}[n], \mathbf{s}[n], \mathbf{v}[n], \mathbf{a}[n], T[n] \\
\mathbf{f}_{1}[n], \mathbf{f}_{2}[n], \mathbf{f}_{3}[n], P_{\mathrm{P}}[n], \eta_{m}}}: F^{\prime} \\
& \text { s.t. } \quad(13 a)-(13 f),(13 i)-(130) \text {, } \\
& \text { (14a) and (14b). }
\end{aligned}
$$

Notice that constraints (13g) and (13h) are decoupled and all the couplings of problem $\mathrm{P}^{\prime \prime \prime}$ are in inequality constraints now. Therefore, the BCD technique can be used now for problem $\mathrm{P} 1^{\prime \prime \prime}$.

\section{B. The BCD-type Iterative Algorithm}

In this subsection, we propose an efficient iterative algorithm by applying the BCD technique for $\mathrm{P}^{\prime \prime \prime}$ solution. To be specific, optimization variables $\alpha_{m}[n], \mathbf{s}[n], \mathbf{v}[n], \mathbf{a}[n]$, $T[n], \mathbf{f}_{1}[n], \mathbf{f}_{2}[n], \mathbf{f}_{3}[n]$ and $P_{\mathrm{t}}[n]$ of $\mathrm{P}^{\prime \prime \prime}$ are partitioned into four sets and alternately optimized while keeping other sets fixed. Additionally, the optimized variables in each iteration are served as inputs of the next iteration. These four sets includes user scheduling and association set $\mathbf{A}=$ $\left\{\alpha_{m}[n], \forall m, n\right\}$, UAV flying status related parameters set $\mathbf{Q}_{\mathbf{1}}=\left\{\mathbf{s}[n], \mathbf{v}[n], \mathbf{a}[n], \mathbf{f}_{1}[n], \mathbf{f}_{2}[n], \mathbf{f}_{3}[n], \forall n\right\}$, UAV completion time and time in different slots set $\mathbf{Q}_{\mathbf{2}}=\{T[n], \forall n\}$, and UAV transmit power allocation set $\mathbf{P}=\left\{P_{\mathrm{t}}[n], \forall n\right\}$. Meanwhile, we decompose the problem $\mathrm{P} 1^{\prime \prime \prime}$ into four subproblems corresponding to these four sets. Specifically, with given sets $\mathbf{Q}_{\mathbf{1}}, \mathbf{Q}_{\mathbf{2}}$ and $\mathbf{P}$, the first sub-problem can be formulated as

$$
\begin{aligned}
(\mathrm{P} 1.1): & \max _{\left\{\mathbf{A}, \eta_{m}\right\}}: F^{\prime} \\
\text { s.t. } & (13 \mathrm{a})-(13 \mathrm{~d}) .
\end{aligned}
$$

Note that except the two constraints (13b) and (13c), defining binary variables, $\mathrm{P} 1.1$ has a linear objective function, and (13a) and (13d) are both linear constraints. Therefore, P1.1 is a mixed-integer linear problem, which can be addressed efficiently by existing software toolboxs such as Gurobi and MOSEK [9], [11].
Similarly, by fixing sets $\mathbf{A}, \mathbf{Q}_{\mathbf{2}}$ and $\mathbf{P}$, set $\mathbf{Q}_{\mathbf{1}}$ can be optimized by addressing the following sub-problem

$$
\begin{aligned}
(\mathrm{P} 1.2): & \max _{\left\{\mathbf{Q}_{1}, \eta_{m}\right\}}: F^{\prime} \\
\text { s.t. } & (13 \mathrm{a}),(13 \mathrm{~b}),(13 \mathrm{e}),(13 \mathrm{f}),(13 \mathrm{i})-(13 \mathrm{~m}), \\
& (14 \mathrm{a}) \text { and }(14 \mathrm{~b}) .
\end{aligned}
$$

Note that constraints (13f), (13i), (14a) and (14b) are linear, (13j), (13l) and (13m) are convex and (13b) specifies that $\eta_{m}$ is a binary variable ${ }^{3}$. So the difficulty of solving P1.2 lies in constraints (13a), (13e) and (13k), which are all non-convex.

First, constraint (13a) can be handled with the SCA technique by deriving its global lower bound at any given point [27]. Specifically, the left-hand-side (LHS) of (13a) is convex with respect to $\left\|\mathbf{s}[n]-\mathbf{w}_{m}\right\|^{2}{ }^{4}$. By applying the fact that the first-order Taylor expansion is the global lower bound of any convex function, thus we find the lower bound $R_{m}^{\mathrm{lb}}$ for $R_{m}$ at any given UAV locations in (17) shown in the top of next page, with $\mathbf{s}^{(l)}[n]$ is the UAV location given by $l$-th iteration. The equality of (17) holds at $\mathbf{s}[n]=\mathbf{s}^{(l)}[n]$.

Furthermore, for addressing the non-convexity of (13e) and (13k), we introduce slack variables $\left\{\tau_{n}\right\}$ [19], [20] and reformulate (13e) and (13k) as

$$
\begin{aligned}
& \sum_{n=1}^{N}\left(c_{1}\|\mathbf{v}[n]\|^{3}+\frac{c_{2}}{\tau_{n}}\left(1+\frac{\|\mathbf{a}[n]\|^{2}}{G^{2}}\right)\right) T[n] \\
& +\sum_{n=1}^{N} P_{\mathrm{t}}[n] T[n] \leq E_{\mathrm{tot}}, \\
& \tau_{n} \geq v_{\min }, \forall n, \\
& \|\mathbf{v}[n]\|^{2} \geq \tau_{n}{ }^{2}, \forall n .
\end{aligned}
$$

With $\tau_{n}$, variables $\mathbf{v}[n]$ and $\mathbf{a}[n]$ are no more coupled in (18), and the LHS of (18) is now jointly convex with respect to $\tau_{n}, \mathbf{v}[n]$ and $\mathbf{a}[n]$. It can be shown that at the optimal solution to P1.2, we must have $\|\mathbf{v}[n]\|=\tau_{n}$. However, the constraint (20) uses an inequality replacing $\|\mathbf{v}[n]\|=\tau_{n}$. Now, we prove that the problem P1.2 with (13e), (13k) and P1.2 with (18)-(20) still keeps the equivalence. Suppose that the optimal solution to P1.2 with (18)-(20) does not satisfy $\|\mathbf{v}[n]\|=\tau_{n}$. Then one can appropriately increase $\tau_{n}$ to make $\|\mathbf{v}[n]\|=\tau_{n}$ hold, which reduces the consumed energy in (18) and results in an increase of the objective value. Therefore, at the optimal solution to P1.2 with (18)-(20), all constraints in (20) must be satisfied with equality.

By replacing (13e) and (13k) with (18)-(20), P1.2 is still non-convex due to the new introduced non-convex constraint

\footnotetext{
${ }^{3}$ We relax the binary variable $\eta_{m}$ into continuous variable, and rewrite the constraint (13b) as $0 \leq \eta_{m} \leq 1, \forall m$.

${ }^{4}$ Note that function $h(x) \triangleq \log \left(1+\frac{C_{1}}{x+C_{2}}\right)$ is convex for constant $C_{1}$ and $C_{2}$ when $C_{1} \geq 0$ and $x+C_{2} \geq 0$. The convexity of $h(x)$ can be easily verified by its second-order conditions [31].
} 


$$
\begin{aligned}
F^{\prime}= & \sum_{m \in M} \omega_{m} \eta_{m}-\sum_{n \in N} \frac{1}{2 \rho_{1}[n]}\left\|\mathbf{f}_{1}[n]-\mathbf{v}[n] T[n]+\rho_{1}[n] \boldsymbol{\lambda}_{1}[n]\right\|^{2} \\
& -\sum_{n \in N} \frac{1}{2 \rho_{2}[n]}\left\|\mathbf{f}_{2}[n]-\frac{1}{2} \mathbf{a}[n] T^{2}[n]+\rho_{2}[n] \boldsymbol{\lambda}_{2}[n]\right\|^{2}-\sum_{n \in N} \frac{1}{2 \rho_{3}[n]}\left\|\mathbf{f}_{3}[n]-\mathbf{a}[n] T[n]+\rho_{3}[n] \boldsymbol{\lambda}_{3}[n]\right\|^{2} .
\end{aligned}
$$

$$
\begin{aligned}
& R_{m}=\sum_{n=1}^{N} \alpha_{m}[n] T[n] B \log _{2}\left(1+\frac{P_{\mathrm{t}}[n] \zeta_{0}}{H^{2}+\left\|\mathbf{s}[n]-\mathbf{w}_{m}\right\|^{2}}\right) \\
& \quad \geq \sum_{n=1}^{N} \alpha_{m}[n] T[n] B \cdot C_{m}^{(l)}[n]-\sum_{n=1}^{N} \alpha_{m}[n] T[n] B \cdot D_{m}^{(l)}[n]\left(\left\|\mathbf{s}[n]-\mathbf{w}_{m}\right\|^{2}-\left\|\mathbf{s}^{(l)}[n]-\mathbf{w}_{m}\right\|^{2}\right) \triangleq R_{m}^{\mathrm{lb}}, \\
& \text { where, } C_{m}^{(l)}[n]=\log _{2}\left(1+\frac{P_{\mathrm{t}}[n] \zeta_{0}}{H^{2}+\left\|\mathbf{s}^{(l)}[n]-\mathbf{w}_{m}\right\|^{2}}\right) \\
& \quad D_{m}^{(l)}[n]=\frac{\left(\log _{2} e\right) P_{\mathrm{t}}[n] \zeta_{0}}{\left(H^{2}+\left\|\mathbf{s}^{(l)}[n]-\mathbf{w}_{m}\right\|^{2}\right)\left(H^{2}+\left\|\mathbf{s}^{(l)}[n]-\mathbf{w}_{m}\right\|^{2}+P_{\mathrm{t}}[n] \zeta_{0}\right)} .
\end{aligned}
$$

(20). Similar to the solution to (13a), the SCA technique is applied again for the LHS of (20). Specifically, $\|\mathbf{v}[n]\|^{2}$ can be replaced by its lower-bound obtained with any given local point $\left\{\mathbf{v}^{(l)}[n]\right\}$, where $\mathbf{v}^{(l)}[n]$ is the optimized velocity in $l$ th iteration. By leveraging the first-order Taylor expansion of $\|\mathbf{v}[n]\|^{2}$, we have the following inequality

$$
\begin{aligned}
\|\mathbf{v}[n]\|^{2} & \geq\left\|\mathbf{v}^{(l)}[n]\right\|^{2}+2 \mathbf{v}^{(l)}[n]\left(\mathbf{v}[n]-\mathbf{v}^{(l)}[n]\right)^{T} \\
& \triangleq \psi_{\mathrm{lb}}(\mathbf{v}[n]),
\end{aligned}
$$

where the equality holds at the point $\mathbf{v}[n]=\mathbf{v}^{(l)}[n]$. Since the right-hand-side (RHS) of (20) is linear and the LHS is convex now, the inequality (20) is convex now.

By replacing the non-convex constraints (13a) and (13k) of the original P1.2 with their corresponding lower bounds at the $l$-th iteration obtained above and decoupling (13e) with a slack variable $\tau_{m}$, we have the following optimization problem

$$
\begin{aligned}
\left(\mathrm{P} 1.2^{\prime}\right): & \max _{\mathbf{Q}_{1}, \eta_{m}, \tau_{n}}: F^{\prime} \\
\text { s.t. } \quad & R_{m}^{\mathrm{b}} \geq \eta_{m} Q_{m}, \forall m, \\
& \psi_{\mathrm{lb}}(\mathbf{v}[n]) \geq \tau_{n}{ }^{2}, \forall n, \\
& (13 \mathrm{~b}),(13 \mathrm{f}),(13 \mathrm{i}),(13 \mathrm{j}),(13 \mathrm{l}), \\
& (13 \mathrm{~m}),(14 \mathrm{a}),(14 \mathrm{~b}),(18) \text { and }(19) .
\end{aligned}
$$

Since all constraints of $\mathrm{P} 1.2^{\prime}$ and the objective function are convex or linear now, the optimization problem can be efficiently addressed by standard optimization solvers, ie. 3dpt solver in CVX. Note that due to the global lower bounds in (13a) and (13k) are applied in $\mathrm{P} 1.2^{\prime}$, if the constraints of $\mathrm{P} 1.2^{\prime}$ are guaranteed, then those for the original sub-problem P1.2 are guaranteed as well, but the reverse is not true. Therefore, the feasible region of $\mathrm{P} 1.2^{\prime}$ is a subset of that for $\mathrm{P} 1.2$, and the optimal solution of $\mathrm{P} 1.2^{\prime}$ is in general a lower bound to that of P1.2. Now, by successively updating the set $\mathbf{Q}_{\mathbf{1}}$ in each iteration via solving $\mathrm{P} 1.2^{\prime}$, we can address the non-convex sub-problem P1.2.

Then, with given sets $\mathbf{A}, \mathbf{Q}_{\mathbf{1}}$ and $\mathbf{P}, \mathbf{Q}_{\mathbf{2}}$ can be optimized by solving the following sub-problem

$$
\begin{aligned}
(\mathrm{P} 1.3): & \max _{\mathbf{Q}_{\mathbf{2}}, \eta_{m}}: F^{\prime} \\
\text { s.t. } & (13 \mathrm{a}),(13 \mathrm{~b}),(13 \mathrm{e}) \text { and }(13 \mathrm{n}) .
\end{aligned}
$$

In P1.3, (13a), (13e) and (13n) are convex and (13b) is a binary constraint similar to that of $\mathrm{P} 1.2^{\prime}$. But $\mathrm{P} 1.3$ is still non-convex since the third term of the objective function is non-concave. Similar to apply the SCA technique for addressing constraints (13a) and (13k) in P1.2, this non-concave term can be handled by the SCA technique.

We denote $\mathbf{f}_{2}[n]=\left[f_{2}^{x}[n], f_{2}^{y}[n]\right], \mathbf{a}[n]=\left[a_{x}[n], a_{y}[n]\right]$, $\boldsymbol{\lambda}_{2}[n]=\left[\lambda_{2}^{x}[n], \lambda_{2}^{y}[n]\right]$ and rewrite the third term of the objective function as (23) shown in the top of next page. It can be seen that the first term in the RHS of (23) is fixed in the sub-problem P1.3, and the second term is concave. Thus, the concavity of the objective function of P1.3 depends on the last term of the RHS of (23). And the concavity of $J[n] T^{2}[n]$ relies on $J[n]$. If $J[n] \leq 0$, we denote $n \in \mathbf{N}_{1}$ and $J[n] T^{2}[n]$ is concave. Else if $J[n]>0$, we denote $n \in \mathbf{N}_{2}$ and a lower-bound of $J[n] T^{2}[n]$ can be obtained with any given local point $T^{(l)}[n]$ by leveraging the first-order Taylor expansion of $J[n] T^{2}[n]$. We then have the following inequality

$$
J[n] T^{2}[n] \geq J[n]\left[\left(T^{(l)}[n]\right)+2 T^{(l)}[n]\left(T[n]-T^{(l)}[n]\right)\right] .
$$

Thus, $F^{\prime}$ of sub-problem P1.3 is rewritten as (25) shown in the top of next page, and $\mathrm{P} 1.3^{\prime}$ is reformulated as

$$
\begin{aligned}
\left(\mathrm{P} 1.3^{\prime}\right): & \max _{\mathbf{Q}_{\mathbf{2}}, \eta_{m}}: F^{\prime \prime} \\
\text { s.t. } & (13 \mathrm{a}),(13 \mathrm{~b}),(13 \mathrm{e}) \text { and (13n). }
\end{aligned}
$$

It is verified that sub-problem P1.3' is a convex optimization problem, that can be efficiently solved as well. Note that due to the global lower bounds in the objective function of $\mathrm{P} 1.3^{\prime}$, 


$$
\begin{aligned}
-\sum_{n \in N} \frac{1}{2 \rho_{2}[n]}\left\|\mathbf{f}_{2}[n]-\frac{1}{2} \mathbf{a}[n] T^{2}[n]+\rho_{2}[n] \boldsymbol{\lambda}_{2}[n]\right\|^{2}= & -\sum_{n \in N} \frac{1}{2 \rho_{2}[n]}\left[\left(f_{2}^{x}[n]+\rho_{2}[n] \lambda_{2}^{x}[n]\right)^{2}+\left(f_{2}^{y}[n]+\rho_{2}[n] \lambda_{2}^{y}[n]\right)^{2}\right] \\
& -\sum_{n \in N} \frac{1}{2 \rho_{2}[n]} \cdot \frac{1}{4}\left(\left(a_{x}[n]\right)^{2}+\left(a_{y}[n]\right)^{2}\right) T^{4}[n]+\sum_{n \in N} \frac{1}{2 \rho_{2}[n]} J[n] T^{2}[n]
\end{aligned}
$$

$$
\text { where, } J[n]=a_{x}[n]\left(f_{2}^{x}[n]+\rho_{2}[n] \lambda_{2}^{x}\right)+a_{y}[n]\left(f_{2}^{y}[n]+\rho_{2}[n] \lambda_{2}^{y}\right) \text {. }
$$

$$
\begin{aligned}
F^{\prime \prime}= & \sum_{m \in M} \omega_{m} \eta_{m}-\sum_{n \in N} \frac{1}{2 \rho_{1}[n]}\left\|\mathbf{f}_{1}[n]-\mathbf{v}[n] T[n]+\rho_{1}[n] \boldsymbol{\lambda}_{1}[n]\right\|^{2} \\
& -\sum_{n \in N} \frac{1}{2 \rho_{2}[n]}\left[\left(f_{2}^{x}[n]+\rho_{2}[n] \lambda_{2}^{x}[n]\right)^{2}+\left(f_{2}^{y}[n]+\rho_{2}[n] \lambda_{2}^{y}[n]\right)^{2}\right]^{2} \\
& -\sum_{n \in N} \frac{1}{8 \rho_{2}[n]}\left(\left(a_{x}[n]\right)^{2}+\left(a_{y}[n]\right)^{2}\right) T^{4}[n]+\sum_{n \in N_{1}} \frac{1}{2 \rho_{2}[n]} J[n] T^{2}[n] \\
& +\sum_{n \in N_{2}} \frac{1}{2 \rho_{2}[n]} J[n]\left[\left(T^{(l)}[n]\right)^{2}+2 T^{(l)}[n]\left(T[n]-T^{(l)}[n]\right)\right] \\
& -\sum_{n \in N} \frac{1}{2 \rho_{3}[n]}\left\|\mathbf{f}_{3}[n]-\mathbf{a}[n] T[n]+\rho_{3}[n] \boldsymbol{\lambda}_{3}[n]\right\|^{2} .
\end{aligned}
$$

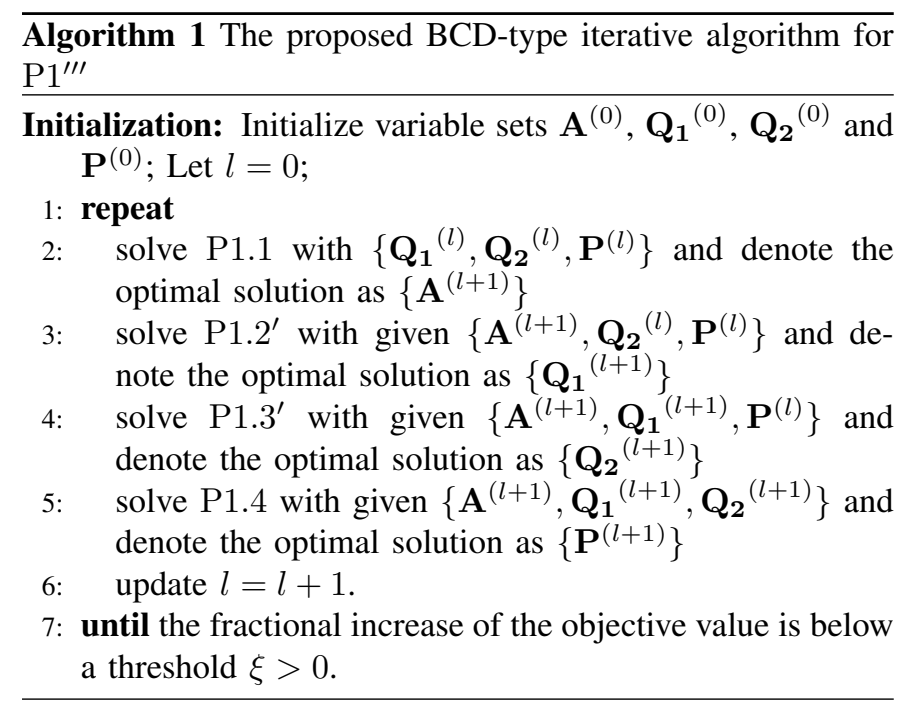

the optimal value of $\mathrm{P} 1.3^{\prime}$ is in general a lower bound to that of P1.3. Now, by successively updating the set $\mathbf{Q}_{\mathbf{2}}$ at each iteration via solving $\mathrm{P} 1.3^{\prime}$, we can address the non-convex sub-problem P1.3.

Finally, by given sets $\mathbf{A}, \mathbf{Q}_{\mathbf{1}}$ and $\mathbf{Q}_{\mathbf{2}}$, the transmit power set $\mathbf{P}$ can be optimized by sub-problem $\mathrm{P} 1.4$ as

$$
\begin{aligned}
(\mathrm{P} 1.4): & \max _{\mathbf{P}, \eta_{m}}: F^{\prime} \\
\text { s.t. } & (13 \mathrm{a}),(13 \mathrm{~b}),(13 \mathrm{e}) \text { and }(13 \mathrm{o}) .
\end{aligned}
$$

Note that P1.4 is convex which can be addressed directly.

Based on the solution of four sub-problems P1.1, P1.2', $\mathrm{P} 1.3^{\prime}$ and $\mathrm{P} 1.4, \mathrm{P}^{\prime \prime \prime}$ can be addressed now. By solving these sub-problems alternatively in each iteration, the BCD-type iterative algorithm is proposed. For simplicity, the iterative algorithm is summarized in Algorithm 1. Additionally, the iterative process will stop when the fractional increase of the objective value is below a threshold. For saving the computation time, in the simulation process in this paper, we set the maximum iteration times for Algorithm 1 is 5 .

In the following, we prove the convergence of Algorithm 1. Define $\Theta\left(\mathbf{A}^{(l)}, \mathbf{Q}_{\mathbf{1}}{ }^{(l)}, \mathbf{Q}_{\mathbf{2}}{ }^{(l)}, \mathbf{P}^{(l)}\right)$ as the objective value of $\mathrm{P} 1^{\prime \prime \prime}, \Theta_{1}^{\mathrm{lb}}\left(\mathbf{A}^{(l)}, \mathbf{Q}_{\mathbf{1}}{ }^{(l)}, \mathbf{Q}_{\mathbf{2}}{ }^{(l)}, \mathbf{P}^{(l)}\right)$ as the objective value of $\mathrm{P} 1.2^{\prime}$, and $\Theta_{2}^{\mathrm{lb}}\left(\mathbf{A}^{(l)}, \mathbf{Q}_{\mathbf{1}}{ }^{(l)}, \mathbf{Q}_{\mathbf{2}}{ }^{(l)}, \mathbf{P}^{(l)}\right)$ as the objective value of $\mathrm{P} 1.3^{\prime}$. It then follows that

$$
\begin{aligned}
& \Theta\left(\mathbf{A}^{(l)}, \mathbf{Q}_{\mathbf{1}}{ }^{(l)}, \mathbf{Q}_{\mathbf{2}}{ }^{(l)}, \mathbf{P}^{(l)}\right) \\
& \leq \Theta\left(\mathbf{A}^{(l+1)}, \mathbf{Q}_{\mathbf{1}}{ }^{(l)}, \mathbf{Q}_{\mathbf{2}}{ }^{(l)}, \mathbf{P}^{(l)}\right) \\
& \stackrel{b}{=} \Theta_{1}^{\mathrm{lb}}\left(\mathbf{A}^{(l+1)}, \mathbf{Q}_{\mathbf{1}}{ }^{(l)}, \mathbf{Q}_{\mathbf{2}}{ }^{(l)}, \mathbf{P}^{(l)}\right) \\
& \stackrel{c}{\leq} \Theta_{1}^{\mathrm{lb}}\left(\mathbf{A}^{(l+1)}, \mathbf{Q}_{\mathbf{1}}{ }^{(l+1)}, \mathbf{Q}_{\mathbf{2}}{ }^{(l)}, \mathbf{P}^{(l)}\right) \\
& \stackrel{d}{\leq} \Theta\left(\mathbf{A}^{(l+1)}, \mathbf{Q}_{\mathbf{1}}{ }^{(l+1)}, \mathbf{Q}_{\mathbf{2}}{ }^{(l)}, \mathbf{P}^{(l)}\right) \\
& \stackrel{e}{=} \Theta_{2}^{\mathrm{lb}}\left(\mathbf{A}^{(l+1)}, \mathbf{Q}_{\mathbf{1}}{ }^{(l+1)}, \mathbf{Q}_{\mathbf{2}}{ }^{(l)}, \mathbf{P}^{(l)}\right) \\
& \stackrel{f}{\leq} \Theta_{2}^{\mathrm{lb}}\left(\mathbf{A}^{(l+1)}, \mathbf{Q}_{\mathbf{1}}{ }^{(l+1)}, \mathbf{Q}_{\mathbf{2}}{ }^{(l+1)}, \mathbf{P}^{(l)}\right) \\
& \stackrel{g}{\leq} \Theta\left(\mathbf{A}^{(l+1)}, \mathbf{Q}_{\mathbf{1}}{ }^{(l+1)}, \mathbf{Q}_{\mathbf{2}}{ }^{(l+1)}, \mathbf{P}^{(l)}\right) \\
& \frac{h}{\leq} \Theta\left(\mathbf{A}^{(l+1)}, \mathbf{Q}_{\mathbf{1}}{ }^{(l+1)}, \mathbf{Q}_{\mathbf{2}}{ }^{(l+1)}, \mathbf{P}^{(l+1)}\right),
\end{aligned}
$$

where (a) holds since in step 2 of Algorithm 1, the optimal solution of P1.1 is obtained based on given $\mathbf{Q}_{\mathbf{1}}{ }^{(l)}, \mathbf{Q}_{\mathbf{2}}{ }^{(l)}$ and $\mathbf{P}^{(l)}$; (b) holds since the first-order Taylor expansions in (17) and (21) are tight at given $\mathbf{Q}_{\mathbf{1}}{ }^{(l)}, \mathbf{Q}_{\mathbf{2}}{ }^{(l)}$ and $\mathbf{P}^{(l)}$, so P1.2 and $\mathrm{P} 1.2^{\prime}$ have the identical objective value; (c) holds since with 


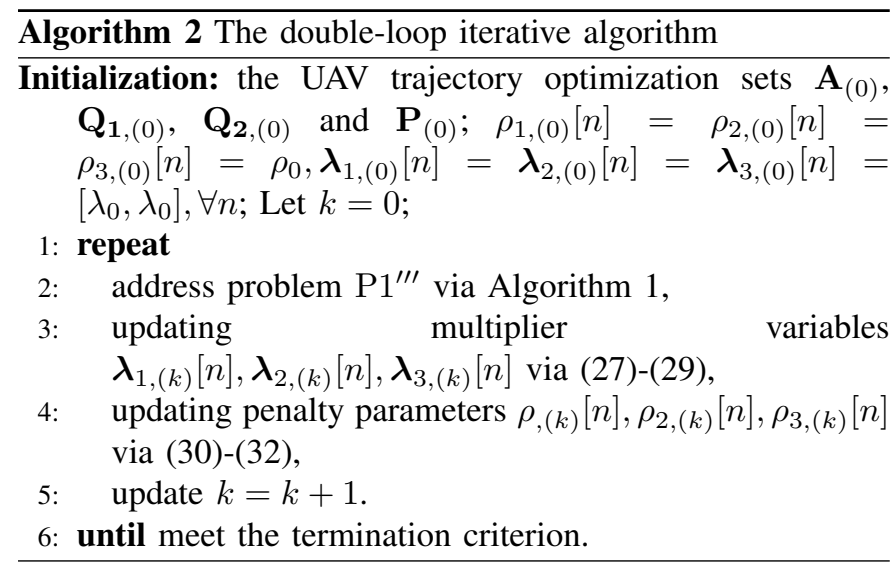

the given $\mathbf{A}^{(l+1)}, \mathbf{Q}_{\mathbf{2}}{ }^{(l)}$ and $\mathbf{P}^{(l)}, \mathrm{P} 1.2^{\prime}$ is optimally addressed in step 3 of Algorithm 1 with solution $\mathbf{Q}_{\mathbf{1}}{ }^{(l+1)}$; (d) holds as the objective value obtained by solving $\mathrm{P} 1.2^{\prime}$ serves as the lowerbound of the original problem P1.2; (e) holds since P1.3 and $\mathrm{P} 1.3^{\prime}$ have the identical objective value since the first-order Taylor expansions in the objective function are tight with given $\mathbf{A}^{(l+1)}, \mathbf{Q}_{\mathbf{1}}{ }^{(l+1)}$ and $\mathbf{P}^{(l)}$; (f) holds since $\mathrm{P} 1.3^{\prime}$ is optimally addressed; (g) holds as the objective value obtained by solving P1.3' serves as the lower-bound of P1.3; (h) holds since in step 5 of Algorithm 1, the optimal solution of P1.4 is obtained based on given $\mathbf{A}^{(l+1)}, \mathbf{Q}_{\mathbf{1}}{ }^{(l+1)}$ and $\mathbf{Q}_{\mathbf{2}}{ }^{(l+1)}$. Therefore, (26) suggests that the proposed algorithm is non-decreasing.

\section{The Proposed Iterative Algorithm}

In the subsection $\mathrm{B}$, an iterative algorithm is applied for addressing $\mathrm{P} 1^{\prime \prime \prime}$ with the given values of the penalty method . However, the penalty method is also an iterative process which need to update its own $\boldsymbol{\lambda}_{1}[n], \boldsymbol{\lambda}_{2}[n], \boldsymbol{\lambda}_{3}[n], \rho_{1}[n]$, $\rho_{2}[n]$ and $\rho_{3}[n]$. Therefore, the overall proposed algorithm will be a double-loop iterative process. The inner iteration is employed for UAV trajectory design based on the BCD technique and the outer iteration is used to update the penalty method. In the outer iterative process, the multiplier variables $\boldsymbol{\lambda}_{1}[n], \boldsymbol{\lambda}_{2}[n], \boldsymbol{\lambda}_{3}[n]$ and the penalty parameters $\rho_{1}[n], \rho_{2}[n]$, $\rho_{3}[n]$ are updated. The multiplier variables are updated as [25], [26]

$$
\begin{aligned}
& \boldsymbol{\lambda}_{1,(k+1)}[n]=\boldsymbol{\lambda}_{1,(k)}[n]+\frac{\left(\mathbf{f}_{1,(k)}[n]-\mathbf{v}_{(k)}[n] T_{(k)}[n]\right)}{\rho_{1,(k)}[n]}, \forall n, \\
& \boldsymbol{\lambda}_{2,(k+1)}[n]=\boldsymbol{\lambda}_{2,(k)}[n]+\frac{\left(\mathbf{f}_{2,(k)}[n]-\frac{1}{2} \mathbf{a}_{(k)}[n] T_{(k)}^{2}[n]\right)}{\rho_{2,(k)}[n]}, \forall n, \\
& \boldsymbol{\lambda}_{3,(k+1)}[n]=\boldsymbol{\lambda}_{3,(k)}[n]+\frac{\left(\mathbf{f}_{3,(k)}[n]-\mathbf{a}_{(k)}[n] T_{(k)}[n]\right)}{\rho_{3,(k)}[n]},
\end{aligned}
$$

where $\mathbf{f}_{1,(k)}[n], \mathbf{f}_{2,(k)}[n], \mathbf{f}_{3,(k)}[n], \mathbf{v}_{(k)}[n], \mathbf{a}_{(k)}[n]$ and $T_{(k)}[n]$ denote the optimal solution of $\mathbf{f}_{1}[n], \mathbf{f}_{2}[n], \mathbf{f}_{3}[n], \mathbf{v}[n], \mathbf{a}[n]$ and $T[n]$ in $k$-th outer-loop iteration. The penalty parameters are updated as (30)-(32) shown in the top of next page [25], [26].

The double-loop iterative algorithm is summarized in Algorithm 2. Specifically, it consists several steps. Firstly, the optimization variables will be initialized via the initial trajectory, which is introduced in Section IV. Meanwhile, we set $\rho_{1,(0)}[n], \rho_{2,(0)}[n]$ and $\rho_{3,(0)}[n]$ with a same value $\rho_{0}$, i.e., 0.9 , and $\boldsymbol{\lambda}_{1,(0)}[n], \boldsymbol{\lambda}_{2,(0)}[n]$ and $\boldsymbol{\lambda}_{3,(0)}[n]$ with a same vector $\left[\lambda_{0}, \lambda_{0}\right]$, i.e., $[40,40]$. Secondly, with fixed values of the penalty method, $\mathrm{P} 1^{\prime \prime \prime}$ is addressed via Algorithm 1. Then, the penalty method is updated. Finally, update the outer iterative process with $k=k+1$. The double-loop iteration is terminated when the criterion is met, i.e., $\left\|\mathbf{f}_{1,(k)}[n]-\mathbf{v}_{(k)}[n] T_{(k)}[n]\right\|<$ $\delta, \quad\left\|\mathbf{f}_{2,(k)}[n]-\frac{1}{2} \mathbf{a}_{(k)}[n] T_{(k)}^{2}[n]\right\|<\delta \quad<\quad$ and $\left\|\mathbf{f}_{3,(k)}[n]-\mathbf{a}_{(k)}[n] T_{(k)}[n]\right\|<\delta, \forall n$, where $\delta$ is a small positive real number.

\section{INITIAL TRAJECTORY DESign: CiRCUlaR VS TAILORED PATH}

The prerequisite for applying the proposed algorithm is feeding a feasible initial trajectory sets $\mathbf{Q}_{\mathbf{1}}$ and $\mathbf{Q}_{\mathbf{2}}$ into the system. Note that due to the existence of a large number of constraints regarding velocity, acceleration and energy, it is not straightforward to find a feasible initial trajectory which satisfies all the constraints. Additionally, according to [32], [33], both the converged solution and the performance of such iterative algorithm depend on the initialization schemes. In this section, we first design a feasible circular initial trajectory which is simple to implement. Then, for achieving better user coverage performance, we further devise another initial trajectory which offers relatively fair association opportunity to ground users. Since we assume the charging base as $\mathbf{s}_{0}=\left[\frac{L_{\mathrm{s}}}{2}, \frac{L_{\mathrm{s}}}{2}\right]$, which is mentioned in Section II. Therefore, for the initial trajectory, both the start point and the end point are the charging point. After a dispatch, the UAV will back and be recharged at the charging base and then start for another service fly.

\section{A. Circular Initial Trajectory (CIT)}

The typical initial trajectory for our scenario is a circular trajectory [14], [34]. However, the effect of limited on-board energy has not been studied in the related works. It is thus important to devise a new feasible trajectory which satisfies the additional energy and acceleration constraints. In this subsection, we propose a low-complexity initial trajectory based on the simple circular trajectory. For ease of exposition, the designed circular initial trajectory (CIT) can be decomposed into three parts, which are denoted by $\zeta_{1}, \zeta_{2}$ and $\zeta_{3}$ respectively. Specifically, $\zeta_{2}$ is a circular trajectory, where the circle center is the location of the charging base and the radius is set as $r_{\mathrm{t}}=\frac{L_{\mathrm{s}}}{4}$, so the number of users inside and outside the circle is balanced. Additionally, $\zeta_{1}$ represents the path from charging base to $\zeta_{2}$ and is set to be a semicircle with radius $r_{\mathrm{s}}=\frac{r_{\mathrm{t}}}{2}$ and center $\mathbf{c}_{\mathrm{s}}=\mathbf{s}_{0}+\left[r_{\mathrm{s}}, 0\right]^{T}$. Furthermore, $\zeta_{3}$ and $\zeta_{1}$ are symmetric with respect to $\mathbf{c}_{\mathrm{s}}$, so $\zeta_{3}$ is another semicircular trajectory with the same center and radius. As can be seen in Fig. 2, the dispatched UAV first flies along $\zeta_{1}$ represented by the red solid line and meets $\zeta_{2}$ represented by the blue dashed line at cross point $\mathbf{s}_{\mathrm{c}}$, where $\mathbf{s}_{\mathrm{c}}=\mathbf{s}_{0}+\left[r_{\mathrm{t}}, 0\right]^{T}$. After flying along the large circle, the UAV returns to the cross point and 


$$
\begin{gathered}
\rho_{1,(k+1)}[n]= \begin{cases}\beta \cdot \rho_{1,(k)}[n], & \text { if }\left\|\mathbf{f}_{1,(k)}[n]-\mathbf{v}_{(k)}[n] T_{(k)}[n]\right\|^{2}>\gamma\left\|\mathbf{f}_{1,(k-1)}[n]-\mathbf{v}_{(k-1)}[n] T_{(k-1)}[n]\right\|^{2}, \\
\rho_{1,(k)}[n], & \text { otherwise, }\end{cases} \\
\rho_{2,(k+1)}[n]= \begin{cases}\beta \cdot \rho_{2,(k)}[n], & \text { if }\left\|\mathbf{f}_{2,(k)}[n]-\frac{1}{2} \mathbf{a}_{(k)}[n] T_{(k)}^{2}[n]\right\|^{2}>\gamma\left\|\mathbf{f}_{2,(k-1)}[n]-\frac{1}{2} \mathbf{a}_{(k-1)}[n] T_{(k-1)}^{2}[n]\right\|^{2}, \\
\rho_{2,(k)}[n], & \text { otherwise, }\end{cases} \\
\rho_{3,(k+1)}[n]= \begin{cases}\beta \cdot \rho_{3,(k)}[n], & \text { if }\left\|\mathbf{f}_{3,(k)}[n]-\mathbf{a}_{(k)}[n] T_{(k)}[n]\right\|^{2}>\gamma\left\|\mathbf{f}_{3,(k-1)}[n]-\mathbf{a}_{(k-1)}[n] T_{(k-1)}[n]\right\|^{2}, \\
\rho_{3,(k)}[n], & \text { otherwise. }\end{cases}
\end{gathered}
$$

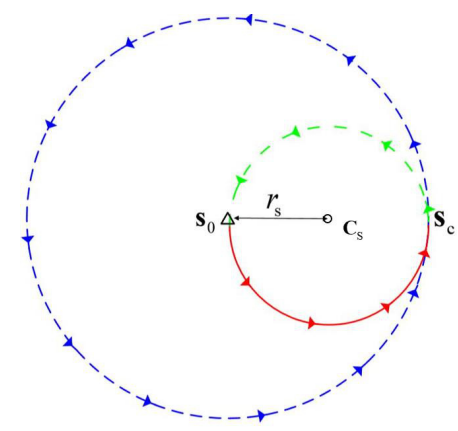

Fig. 2. Illustration of circular initial trajectory.

follows the trajectory of the other semicircle $\zeta_{3}$ until arriving at the charging base.

The UAV flies with constant flying speed $\|\mathbf{v}[n]\|=V$, where $V_{\min } \leq V \leq V_{\max }$. In other words, the UAV follows uniform circular motion with respect to two different circle centers. Note that the value of $V$ should be chosen appropriately such that the acceleration constraint is satisfied. It is known that uniform circular motion leads to an acceleration with constant value $\|\mathbf{a}[n]\|=\frac{V^{2}}{r}$, where $r$ denotes the radius of circular trajectory. However, due to the application of path discretization technique, the resulting trajectory consists of $N$ line segments with same length and forms a regular polygon which is less smooth than circle. This requires sharper direction change and thus larger acceleration at each path slot. Since both velocity and acceleration are vectors, the relationship between $\mathbf{v}[n]$ and $\mathbf{a}[n]$, which is shown in (13h), can also be depicted by the geometrical interactions of two vectors as in Fig. 3, where $\theta$ measures the direction change. We first note that the sum of the interior angles of a polygon with $n$ sides is $(n-2) \cdot \pi$, where $n$ also equals to the number of path slots allocated to the corresponding circle. The number of path slots allocated to the two semicircles can be found by

$$
n_{1}=\frac{2 \pi r_{\mathrm{s}}}{2 \pi r_{\mathrm{s}}+2 \pi r_{\mathrm{t}}} N=\frac{1}{3} N .
$$

Correspondingly, flying along $\zeta_{2}$ takes $n_{2}=\frac{2}{3} N$ path slots. Thus, the direction change between adjacent path slots for the semicircular trajectory and the circular trajectory are computed as $\theta_{1}=\frac{n_{1}-2}{n_{1}} \pi$ and $\theta_{2}=\frac{n_{2}-2}{n_{2}} \pi$ respectively. We initialize that the total completion time is $T_{\text {tot }}$ in CIT. And $T_{\text {tot }}$ is divided into $N$ equal slots which are denoted as $T_{n}$. Since $\|\mathbf{v}[n]\|=\|\mathbf{v}[n+1]\|$, we can find the relationship between $V$

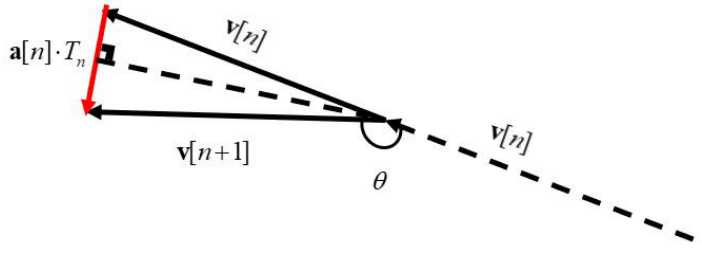

Fig. 3. Geometrical representation of constraint (13h).

and $\|\mathbf{a}[n]\|$ through the geometrical relationship as follows

$$
V=\frac{\|\mathbf{a}[n]\| \cdot T_{n}}{2 \sin \left(\frac{\pi-\theta}{2}\right)}, 0<\theta<\pi .
$$

Since more path slots are allocated to $\zeta_{2}$ compared to $\zeta_{1}$ and $\zeta_{3}$, we have larger $\theta$ value for $\zeta_{2}$. Furthermore, (34) indicates that the value of $V$ increases monotonously with respect to $\theta$, so flying along $\zeta_{2}$ requires smaller acceleration than the two semicircular trajectories with a given velocity. Therefore, by letting $\|\mathbf{a}[n]\|=a_{\max }$, the resulting maximum velocity which satisfies the acceleration constraints is computed as

$$
V_{\max }^{a}=\frac{a_{\max } T_{n}}{2 \sin \left(\frac{\pi-\theta_{1}}{2}\right)} .
$$

In addition to satisfy the acceleration constraints, UAV flying along the proposed CIT should consume less energy than the total on-board energy. By letting $\mathbf{a}[n]=a_{\max }$ and $P_{\mathrm{t}}[n]=P_{0}$, where $P_{0}$ is the original inputs for transmit power, the critical value of $V$ can be found by solving the following equation

$$
\left(c_{1} V^{3}+\frac{c_{2}}{V}\left(1+\frac{a_{\max }^{2}}{G^{2}}\right)+P_{0}\right) \cdot T_{\text {tot }}=E_{\text {tot }},
$$

where (36) has two solutions, which are $V_{\min }^{E}$ and $V_{\max }^{E}$. According to the monotonicity with respect to $V$, all the values within the range $V_{\min }^{E} \leq V \leq V_{\max }^{E}$ are feasible. By applying the parameters shown in Table 1, the consumed energy is dominated by the term $c_{1} V^{3}$, and $V_{\min }^{E}$ is much smaller than $V_{\min }$. Therefore, for satisfying both the acceleration and energy constraints, the velocity of UAV is set as $V_{\mathrm{CIT}}=\min \left(V_{\max }^{E}, V_{\max }^{a}, v_{\max }\right)$.

With $\|\mathbf{v}[n]\|=V_{\mathrm{CIT}}$, the radius of CIT may not be always achievable given the mission period $T_{\text {tot }}$ if $6 \pi r_{\mathrm{s}}>V_{\mathrm{CIT}} T_{\text {tot }}$. Therefore, for guaranteeing that the UAV will return to the charging base with the given time horizon, the radius of 


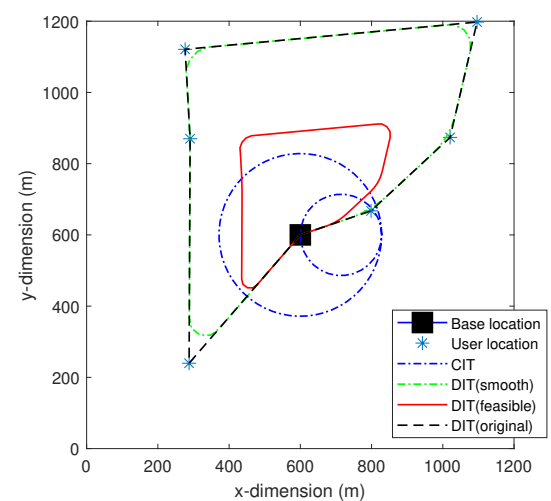

Fig. 4. An example of CIT and DIT, $T_{\text {tot }}=90 \mathrm{~s}, E_{\mathrm{tot}}=5 \mathrm{KJ}$.

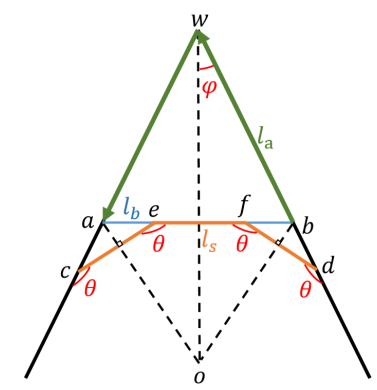

Fig. 5. Trajectory around turning points.

semicircle is set as $r_{\mathrm{cit}}=\min \left(\frac{V_{\mathrm{CIT}} T_{\mathrm{tot}}}{6 \pi}, r_{\mathrm{s}}\right)$. The angle with respect to the circle center is represented as

$$
\Gamma_{n}= \begin{cases}\pi+\pi \frac{n-1}{n_{1}}, & n=1, \ldots, n_{1}, \\ 2 \pi \frac{n-n_{1}-1}{n_{1}-1}, & n=n_{1}+1, \ldots, n_{1}+n_{2}, \\ \pi \frac{n-n_{1}-n_{2}}{N-n_{1}-n_{2}}, & n=n_{1}+n_{2}+1, \ldots, N .\end{cases}
$$

Based on the circle center and radius of CIT, the initial trajectory of UAV in path slot $n$ is then obtained as (38) shown in the top of next page. After obtaining $\mathbf{s}_{0}[n], \mathbf{v}_{0}[n]$ and $\mathbf{a}_{0}[n]$ can be computed.

\section{B. Designed Initial Trajectory (DIT)}

In our specific problem, when the total number of users is small, the optimal objective value is trivial and equals to one. However, when the total number of users is sufficiently large, only part of the ground users can be scheduled and associated. In this case, if CIT is applied to Algorithm 2, users which are closer to the initial trajectory has a higher opportunity to be considered for association due to the lower path loss. Therefore, CIT does not consider fair scheduling and association and may lead to a performance loss. This motivates us to design an initial trajectory which ensures all the ground users can get close to the UAV in certain path slots, so the users have a relatively fair opportunity to be considered for scheduling and association.

To this end, we design an initial trajectory where the UAV flies straightly from one ground user to the other with constant speed $\|\mathbf{v}[n]\|=V$ in the horizontal dimension, and finally backs to the charging base. Specifically, we first convert the location of ground users into polar coordinate system with $\mathbf{s}_{0}$ serves as the coordinate origin and $\mathbf{v}_{f}=\mathbf{s}_{c}-\mathbf{s}_{0}$ serves as the reference direction, that is $\mathbf{w}_{m}^{P}=\left[r_{m}, \phi_{m}\right]$, where $r_{m}=\left\|\mathbf{s}_{0}-\mathbf{w}_{m}\right\|$ and $\phi_{m}=\arctan \left(\frac{y_{m}-\frac{L_{\mathrm{s}}}{2}}{x_{m}-\frac{L_{\mathrm{s}}}{2}}\right) \in(0,2 \pi)$. Starting from the charging base location, DIT is formed by connecting each of the ground users with a line segment based on the size of angle $\phi_{m}$, so user $j$ will be visited before user $m$ if $\phi_{j}<\phi_{m}$. Additionally, if two users have the same angle, the initialization path prioritizes the user which has a smaller $r_{m}$, and the resulting trajectory connecting all user points is shown as DIT (original) in Fig. 4. Note that, the trajectory flying directly from one ground user to the other may incur abrupt direction change at turning points, thus violates the acceleration constraints. For obtaining a feasible DIT, the path around the turning points is designed as a smooth trajectory consisting of three line segments of the same length. As illustrated in Fig. 5, instead of flying along the trajectory which requires a direction change of $2 \varphi$ at point $w$, the UAV follows a more smooth trajectory which is comprised of three line segments $\overline{d f}, \overline{f e}$ and $\overline{e c}$, where $\overline{d f}=\overline{f e}=\overline{e c}=l_{\mathrm{s}}$. With the designed trajectory, moving close to a certain user point requires equal direction change $\theta$ at four points which correspond to point $c$, point $d$, point $e$ and point $f$ in Fig. 5 respectively. The relationship between $\varphi$ and $\theta$ can be derived geometrically as $\theta=\frac{3 \pi}{4}+\frac{\varphi}{2}$. Since $0<\varphi \leq \frac{\pi}{2}$, the direction change between adjacent path slots is tiny and $\theta$ is in the range of $\frac{3 \pi}{4}<\theta \leq \pi$. For applying the designed trajectory, it is imperative to find the location of new turning points. First, with a given $l_{\mathrm{S}}$ which can be chosen as any value larger than $T_{n} v_{\max }$, we find $l_{\mathrm{b}}=\frac{l_{\mathrm{s}}}{2 \sin \left(\frac{\pi}{4}+\frac{\varphi}{2}\right)}$, where $l_{\mathrm{b}}$ is the length of line segments $\overline{a e}, \overline{a c}, \overline{b d}$ and $\overline{b f}$ as shown in Fig. 5. Besides, the position of point $a$ and point $b$ can be found by measuring the value of $l_{\mathrm{a}}$, which denotes the distance between a certain user location and the corresponding point. The value of $l_{\mathrm{a}}$ can be obtained geometrically as

$$
l_{\mathrm{a}}=\frac{\frac{l_{\mathrm{s}}}{2}+l_{\mathrm{b}}}{\sin (\varphi)}=\frac{l_{\mathrm{s}}}{\sin (\varphi)} \cdot \frac{\sin \left(\frac{\pi}{4}+\frac{\varphi}{2}\right)+1}{2 \sin \left(\frac{\pi}{4}+\frac{\varphi}{2}\right)} .
$$

After obtaining $l_{\mathrm{a}}$ and $l_{\mathrm{b}}$, the location of all the new turning points can be easily found. There are a total of $4 M$ turning points, and the location of the $i$-th turning point is denoted as $t_{i}=\left[x_{i}, y_{i}\right]$. We denote by $\theta_{\min }$ the smallest direction change of DIT, which corresponds to the smallest $\varphi$ value. Similar to the case of CIT, the maximum velocity which satisfies the acceleration constraint can be found by substituting $a_{\max }$ and $\theta_{\min }$ into (34) as follows

$$
V_{\max }^{a}=\frac{a_{\max } T_{n}}{2 \sin \left(\frac{\pi-\theta_{\min }}{2}\right)} .
$$

Note that (36) serves as the upper bound of the total consumed energy of a UAV flying along DIT, so the feasible velocity which satisfies all the constraints can also be set as $V_{\text {DIT }}=\min \left(V_{\max }^{E}, V_{\max }^{a}, v_{\max }\right)$, where $V_{\max }^{a}$ takes the value of (40) and $V_{\max }^{E}$ is the solution of (36). The resulting trajectory is shown as DIT (smooth) in Fig. 4.

In order to guarantee that the UAV will return to the charging base with the given time horizon, the total travelling 


$$
\mathbf{s}_{0}[n]= \begin{cases}{\left[\frac{L_{\mathrm{s}}}{2}+r_{\mathrm{s}}+r_{\mathrm{s}} \cos \left(\psi_{n}\right), \frac{L_{\mathrm{s}}}{2}+r_{\mathrm{s}} \sin \left(\psi_{n}\right)\right],} & n=1, \ldots, n_{1}, \\ {\left[\frac{L_{\mathrm{s}}}{2}+r_{\mathrm{t}} \cos \left(\psi_{n}\right), \frac{L_{\mathrm{s}}}{2}+r_{\mathrm{t}} \sin \left(\psi_{n}\right)\right],} & n=n_{1}+1, \ldots, n_{1}+n_{2}, \\ {\left[\frac{L_{\mathrm{s}}}{2}+r_{\mathrm{s}}+r_{\mathrm{s}} \cos \left(\psi_{n}\right), \frac{L_{\mathrm{s}}}{2}+r_{\mathrm{s}} \sin \left(\psi_{n}\right)\right],} & n=n_{1}+n_{2}+1, \ldots, N .\end{cases}
$$

TABLE I

SIMULATION PARAMETERS

\begin{tabular}{|c|c|c|c|}
\hline parameter & value & parameter & value \\
\hline$B$ & $10^{6} \mathrm{~Hz}$ & $H$ & $100 \mathrm{~m}$ \\
\hline$P_{\max }$ & $42 \mathrm{dBm}$ & $G$ & $9.8 \mathrm{~m} / \mathrm{s}^{2}$ \\
\hline$\beta_{0}$ & $-50 \mathrm{~dB}$ & $\Delta$ & $30 \mathrm{~m}$ \\
\hline$\sigma^{2}$ & $-110 \mathrm{dBm}$ & $v_{\max }$ & $60 \mathrm{~m} / \mathrm{s}$ \\
\hline$c_{1}$ & $2 \times 10^{-3}$ & $v_{\min }$ & $3 \mathrm{~m} / \mathrm{s}$ \\
\hline$c_{2}$ & 70.698 & $a_{\max }$ & $5 \mathrm{~m} / \mathrm{s}^{2}$ \\
\hline$P_{0}[n]$ & $0.5 \mathrm{w}$ & $\rho_{0}$ & 0.9 \\
\hline$\lambda_{0}$ & {$[40,40]$} & initial $T_{\text {tot }}$ & $90 \mathrm{~s}$ \\
\hline
\end{tabular}

distance should be no larger than $V_{\mathrm{DIT}} T_{\mathrm{tot}}$. To this end, We further introduce a variable $\lambda_{\text {DIT }}$, with $0<\lambda_{\text {DIT }} \leq 1$, such that

$$
\lambda_{\mathrm{DIT}}= \begin{cases}\frac{V_{\mathrm{DIT}} T_{\mathrm{tot}}}{d_{\mathrm{sum}}}, & \text { if } d_{\mathrm{sum}}>V_{\mathrm{DIT}} T_{\mathrm{tot}}, \\ 1, & \text { if } d_{\mathrm{sum}} \leq V_{\mathrm{DIT}} T_{\mathrm{tot}},\end{cases}
$$

where $d_{\text {sum }}$ denotes the total distance of DIT, and is calculated as

$$
d_{\text {sum }}=\sum_{i=1}^{4 M-1}\left\|\mathbf{t}_{i+1}-\mathbf{t}_{i}\right\|+\left\|\mathbf{s}_{0}-\mathbf{t}_{1}\right\|+\left\|\mathbf{s}_{0}-\mathbf{t}_{4 M}\right\| .
$$

Therefore, the trajectory with a total distance of $\lambda_{\mathrm{DIT}} d_{\text {sum }}$ guarantees that the UAV will return to the base with a given mission period. By applying the property of similar triangle, such a feasible trajectory can be found by connecting each of the $4 M$ points, which are denoted by $\mathbf{t}_{i}^{p}=$ $\left[\lambda_{\text {DIT }}\left\|\mathbf{s}_{0}-\mathbf{t}_{i}\right\|, \phi_{i}=\arctan \left(\frac{y_{i}-\frac{L_{\mathrm{s}}}{2}}{x_{i}-\frac{L_{\mathrm{s}}}{2}}\right) \in(0,2 \pi)\right]$, based on the size of angle $\phi_{i}$. The proposed feasible DIT is also shown in Fig. 4.

For better illustration of the proposed initial trajectory, Fig. 4 also compares CIT and DIT. Note that, the users which are located far away from CIT may never be scheduled and associated by applying Algorithm 2 with CIT due to the large path loss. On the contrary, these users could be served by applying Algorithm 2 with DIT thanks to the significantly reduced transmission distance.

\section{SIMULATION RESULTS AND ANALYSIS}

This section provides numerical results to evaluate the performance of our proposed techniques. We assume $M=6$ users which are distributed randomly within the square target area of $1.2 \times 1.2 \mathrm{~km}^{2}$. Correspondingly, the charging base is located at $[600,600]^{T}$. In addition, the data demand of each user is a random value within the range of $[100,600]$ Mbits. The coverage performance is evaluated with regard to user coverage probability, which is defined as the ratio of the number of served users to the total number of ground users $M$. For ease of presentation, the proposed algorithm in Section III initializing with initial trajectories CIT and DIT are termed as IA-CIT and IA-DIT respectively.

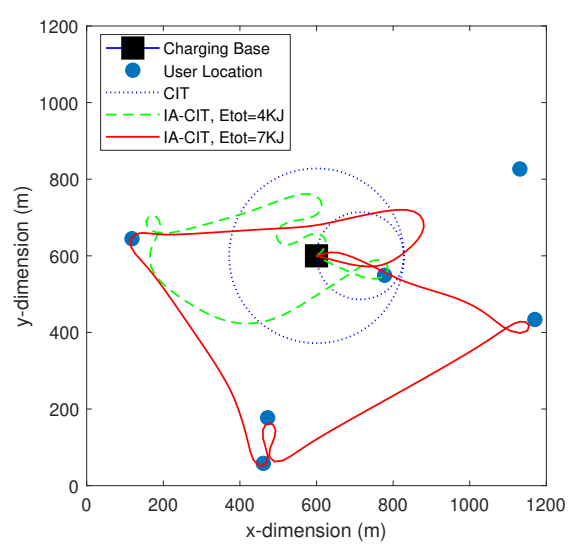

(a) IA-CIT with varying $E_{\text {tot }}$.

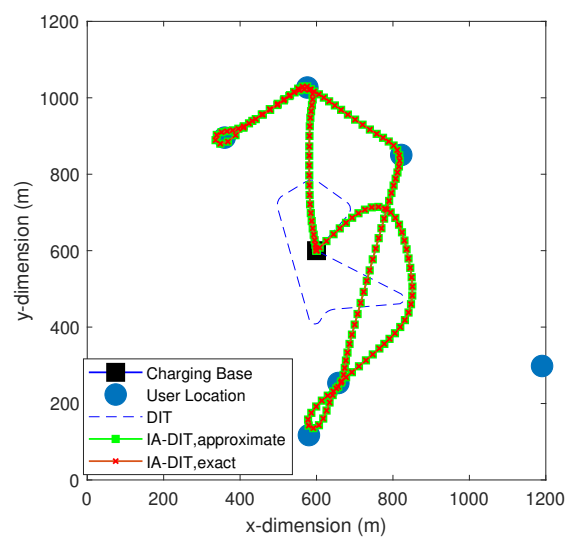

(b) Approximate and exact IA-DITs with $E_{\mathrm{tot}}=6 \mathrm{KJ}$.

Fig. 6. Optimized trajectories.

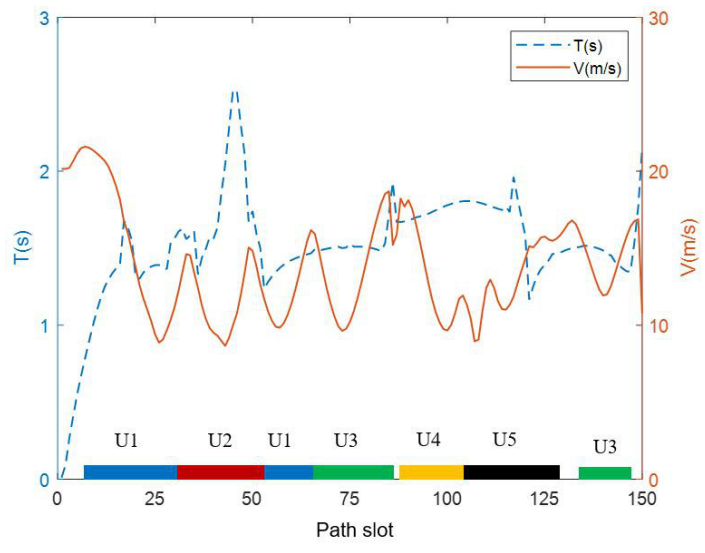

Fig. 7. UAV time and speed corresponding to IA-DIT shown in Fig. 6 (b).

\section{A. Proposed Iterative Algorithm and the Impact of Energy Constraint}

In Fig. 6 (a), we first illustrate optimized trajectories obtained by applying IA-CIT based on different on-board energy 


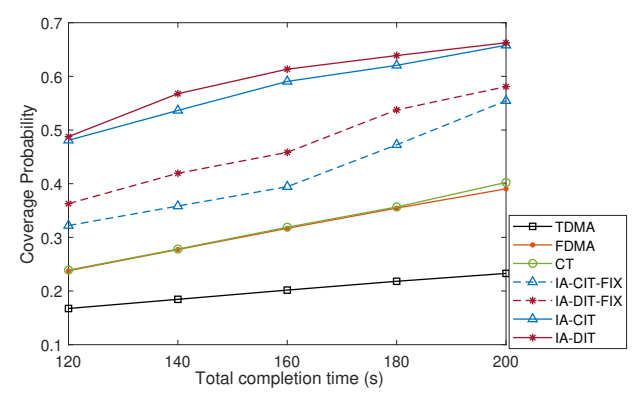

Fig. 8. Coverage probability with different techniques, $E_{\mathrm{tot}}=5 \mathrm{KJ}$.

$E_{\text {tot }}$. Firstly, both these two paths show that the UAV flies close to some ground users when it attempts to serve them. Approaching to these users enables the aerial BS to reduce the communication path loss and thus transmits more data to the corresponding target user. Comparing two IA-CIT trajectories constrained by different $E_{\text {tot }}$, we notice that the UAV can fly closer to all users and can fly a longer distance with more energy. Thus, the UAV can cover more ground users. The UAV trajectories in both Fig. 6 (a) and Fig. 6 (b) are nearly smooth, and this is a result of UAV acceleration constraint, which forbids the UAV to change its direction abruptly.

In Section III, we apply the SCA technique in constraints (13a), (13k) and in the objective function of P1.3. Meanwhile, we exploit some approximations in (14c)-(14e) when decouple the couplings among $\mathbf{v}[n], \mathbf{a}[n]$ and $T[n]$ by applying the penalty method. To evaluate the accuracy of these applied approximations, we illustrate an example about the comparative paths between UAV exact locations and approximate locations. By applying the optimal solution of $\mathbf{a}[n]$ and $T[n]$, we can calculate the UAV exact velocities and then, obtain UAV exact locations and exact path. In Fig. 6 (b), we can find that the exact IA-DIT path and the approximate IA-DIT path from one simulation quite match each other.

Fig. 7 shows the path-varying UAV time and speed as well as the user communication scheduling and association corresponding to the trajectory shown in Fig. 6 (b). It can be seen that the UAV first flies toward the target users with faster speed and less time, then gradually reduces its speed and spends more time when it starts to have a good communication channel with the corresponding users. Since fixed-wing UAV is utilized, the UAV has a minimum speed requirement for maintaining the movement and cannot hover above the served users with zero speed. Note that not all the path slots are allocated for the covered users. This indicates that the UAV tries to allocate redundant path slots to users which cannot be fully served after satisfying the requirement of the covered users.

Fig. 8 compares the achieved coverage probability among seven different schemes, i.e., 1) Static UAV with Time Division Multiple Access (TDMA), where the aerial BS is placed at a location which is 100 meters above the charging base and remains static for the whole service period. In addition, the user communication scheduling and association variables are optimized; 2) Static UAV which is same with 1) but with

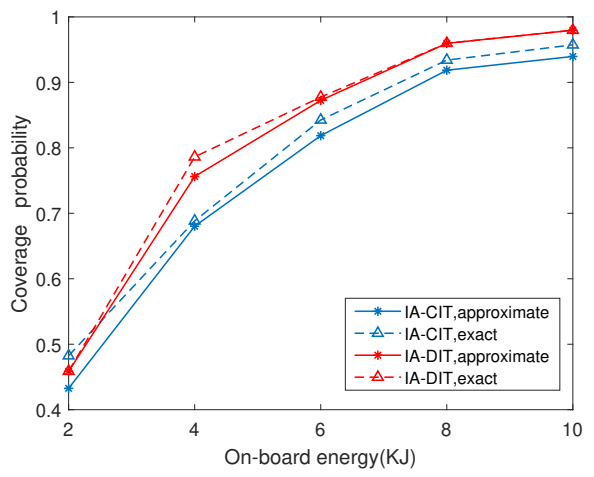

(a) Coverage probability.

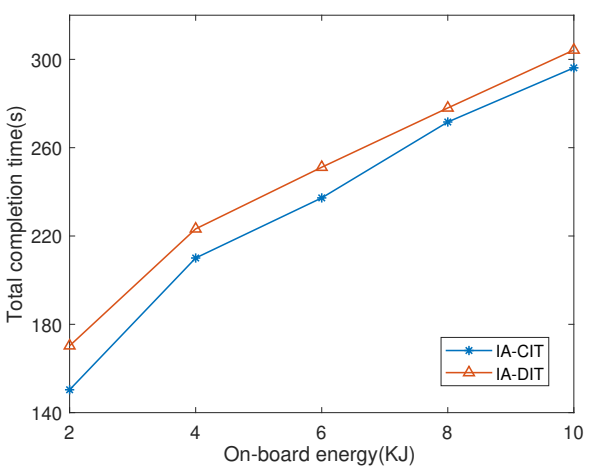

(b) Total completion time.

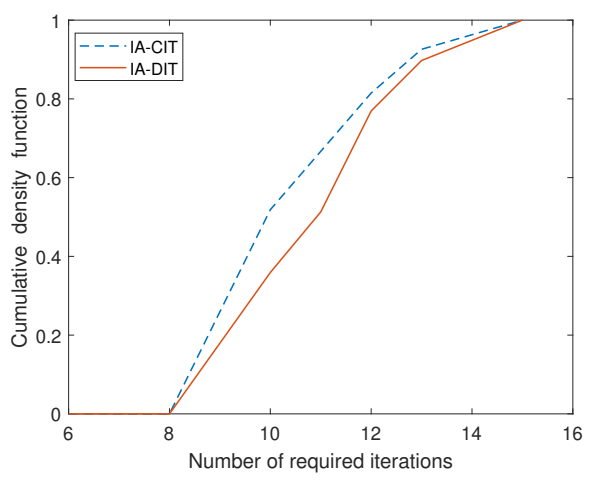

(c) $\mathrm{CDF}, E_{\text {tot }}=5 \mathrm{KJ}$.

Fig. 9. Coverage performance and CDF versus on-board energy $E_{\text {tot }}$ of IA-CIT and IA-DIT.

Frequency Division Multiple Access (FDMA); 3) Circular trajectory (CT), a scheme follows CIT while optimizing user communication scheduling and association, as well as the transmit power; 4) IA-CIT-Fix, which utilizes the proposed algorithm based on CIT but $T[n]$ is pre-determined with $T[n]=\frac{T_{\text {tot }}}{N}$, where $T_{\text {tot }}$ is the total completion time; 5) IADIT-Fix, which is same with 4) but based on DIT; 6) IA-CIT, 7) IA-DIT. Note that, since $T[n]$ is optimized in 6) and 7), to consider about the fairness, the UAV total completion time of IA-CIT and IA-DIT should be no more than $T_{\text {tot }}$ we set for other schemes.

As regards the performance observed, we can first conclude that, by exploiting the UAV mobility, a much better coverage performance can be achieved thanks to the reduced 
communication path loss. Even the CT scheme which has the worst performance among techniques considering moving aerial BSs satisfies more users than static aerial BSs with a larger time. As expected, trajectory based on DIT satisfies a clearly increased number of served users compared to CIT. Finally, the proposed trajectory applying time optimization outperforms the trajectory with fixed $T[n]$. With variable $T[n]$, the trajectory optimization variables can be optimized in a wider range. As such, the UAV can afford more time for data transmission and can reduce the waste of time and energy on the way to approach corresponding users. Therefore, the proposed trajectory outperforms the trajectory with fixed time slots.

A performance comparison between our proposed algorithm based on circular initial trajectory and designed initial trajectory are shown in Fig. 9. In Fig. 9 (a), it can be observed that the coverage of IA-DIT outperforms IA-CIT. Comparing the exact coverage and the optimal solution of $\mathrm{P} 1^{\prime \prime \prime}$, we observe that the two curves match quite well with each other and the obtained optimal solution of $\mathrm{P}^{\prime \prime \prime}$ provides a lower bound for the exact UAV coverage performance. The gains of our energy-constrained trajectory design are tightly related to the on-board battery capacity. We notice that in Fig. 9 (a) and Fig. 9 (b), with the increasing energy, a UAV larger trajectory and completion time is obtained, which enables satisfying the quality of service requirements of more users. Fig. 9 (c) illustrates that the devised initial trajectory requires a higher number of outer-loop iterations for the penalty method. But, the difference about cumulative density function (CDF) between IA-CIT and IA-DIT is very small.

\section{B. Proposed Iterative Algorithm based on Penalty Method Parameters}

In our proposed iterative algorithm based on the penalty method, we set the initial input of multiplier variables as $\boldsymbol{\lambda}_{1,0}[n]=\boldsymbol{\lambda}_{2,0}[n]=\boldsymbol{\lambda}_{3,0}[n]=\left[\lambda_{0}, \lambda_{0}\right]$. In this subsection, we analyze the performance of our proposed algorithm impacted by different $\lambda_{0}$. Fig. 10 (a) illustrates that, we are able to obtain an improvement of UAV coverage probability if we increase the absolute value of $\lambda_{0}$. According to Fig. 10 (c), we realize that with a higher value of $\left|\lambda_{0}\right|, T[n]$ can vary within a wider range among path slots, which means the trajectory optimization is relaxed in a wider range. Accordingly, a higher value of $\left|\lambda_{0}\right|$ results in a higher coverage probability. However, Fig. 10 (b) shows that a higher $\left|\lambda_{0}\right|$ requires a higher number of iterations for the iterative penalty method. Therefore, if we apply the proposed trajectory optimization techniques in practical scenarios, the real ground users demand and the target area should be taken into consideration, in designing the tradeoff between higher coverage performance and less processing time. For example, if the ground scenario requires a quick service from the UAV, a smaller value of $\left|\lambda_{0}\right|$ will be chosen to decrease the processing time for calculating the trajectory, at the expense of a reduced coverage performance.

\section{CONCLUSION}

In this paper, a fixed-wing UAV transmission communication is considered, where an aerial BS is dispatched from

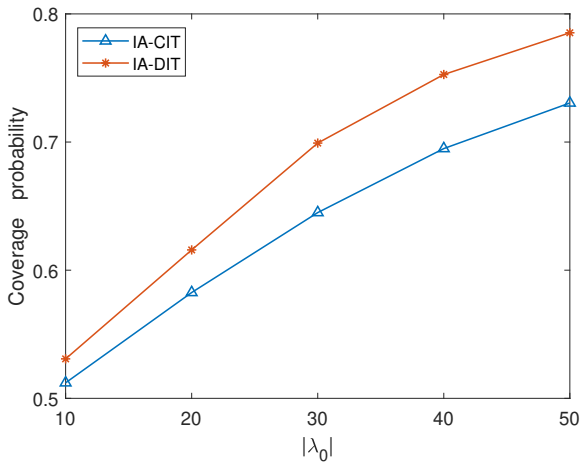

(a) Coverage probability.

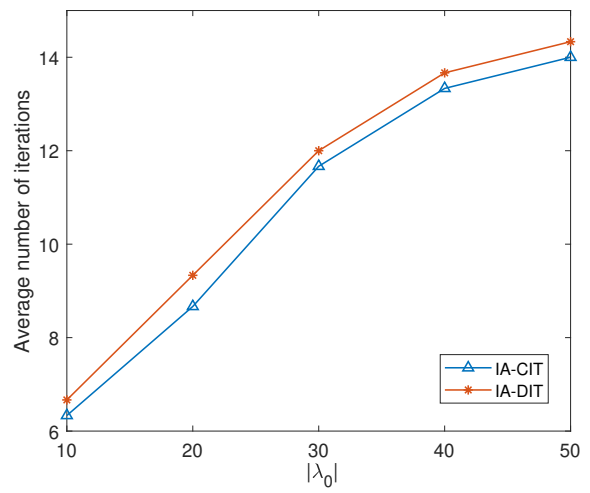

(b) Average number of iterations for the penalty method.

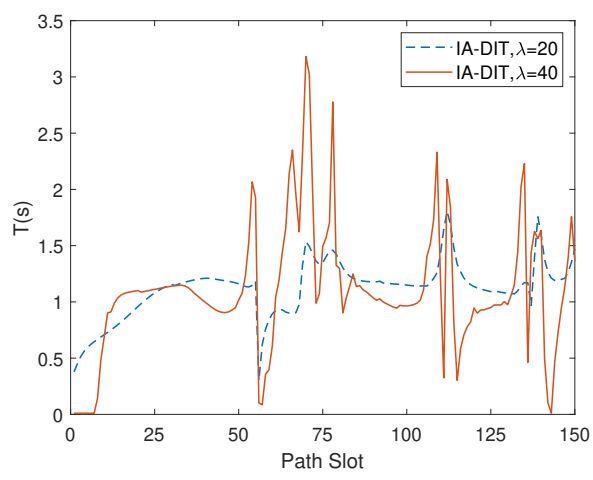

(c) Time corresponding to trajectories with different $\lambda_{0}$.

Fig. 10. Proposed algorithm performance versus different $\left|\lambda_{0}\right|, E_{\text {tot }}=4 \mathrm{KJ}$.

the charging base, flies following an optimized trajectory for covering a maximum number of ground users before exhausting the on-board energy. A double-loop iterative algorithm has been proposed. By applying the penalty method, the difficulties of coulping constraints are addressed. By applying successive convex optimization and block coordinate descent techniques, the iterative algorithm alternately optimizes the user communication scheduling and association, UAV flying status related parameters, UAV completion time, as well as communication time among users, and transmit power in each iteration. In order to achieve a better coverage performance, the initial trajectory is carefully designed so all the ground users have a fair opportunity to be scheduled and associated. Numerical results verifies that the proposed initial trajectory 
results in a performance improvement.

\section{REFERENCES}

[1] M. Mozaffari, W. Saad, M. Bennis, Y. Nam, and M. Debbah, "A tutorial on UAVs for wireless networks: Applications, challenges, and open problems," IEEE Commun. Surveys Tuts., vol. 21, no. 3, pp. 2334-2360, Mar. 2019.

[2] Y. Zeng, R. Zhang, and T. J. Lim, "Wireless communications with unmanned aerial vehicles: Opportunities and challenges," IEEE Commun. Mag., vol. 54, no. 5, pp. 36-42, May 2016.

[3] J. Wang, C. Jiang, Z. Han, Y. Ren, R. G. Maunder, and L. Hanzo, "Taking drones to the next level: Cooperative distributed unmannedaerial-vehicular networks for small and mini drones," IEEE Trans. Veh. Technol., vol. 12, no. 3, pp. 73-82, Sep. 2017.

[4] J. Wang, C. Jiang, Z. Wei, C. Pan, H. Zhang, and Y. Ren, "Joint UAV hovering altitude and power control for space-air-ground IoT networks," IEEE Internet Things J., vol. 6, no. 2, pp. 1741-1753, Apr. 2019.

[5] K. Namuduri, Y. Wan, and M. Gomathisankaran, "Mobile ad hoc networks in the sky: State of the art, opportunities, and challenges," in Proc. 2nd ACM MobiHoc Workshop Airborne Netw. Commun. (ANC), New York, NY, USA, 2013, pp. 25-28.

[6] A. Al-Hourani, S. Kandeepan, and S. Lardner, "Optimal LAP altitude for maximum coverage," IEEE Wireless Commun. Lett., vol. 3, no. 6, pp. 569-572, Dec. 2014.

[7] B. Li, C. Chen, R. Zhang, H. Jiang, and X. Guo, "The energy-efficient UAV-based BS coverage in air-to-ground communications," in Proc. IEEE Sensor Array and Multichannel Signal Processing Workshop (SAM Wkshps), Jul. 2018, pp. 578-581.

[8] M. Mozaffari, W. Saad, M. Bennis, and M. Debbah, "Drone small cells in the clouds: Design, deployment and performance analysis," in Proc. IEEE Global Commun. Conf. (GLOBECOM), Dec. 2015, pp. 1-6

[9] M. Alzenad, A. El-Keyi, F. Lagum, and H. Yanikomeroglu, "3-D placement of an unmanned aerial vehicle base station (UAV-BS) for energy-efficient maximal coverage," IEEE Wireless Commun. Lett., vol. 6, no. 4, pp. 434-437, Aug. 2017.

[10] R. I. Bor-Yaliniz, A. El-Keyi, and H. Yanikomeroglu, "Efficient 3-D placement of an aerial base station in next generation cellular networks," in Proc. IEEE Int. Conf. Commun. (ICC), May 2016, pp. 1-5.

[11] J. Sun and C. Masouros, "Deployment strategies of multiple aerial BSs for user coverage and power efficiency maximization," IEEE Trans. Commun., vol. 67, no. 4, pp. 2981-2994, Apr. 2019.

[12] L. Ruan et al., "Energy-efficient multi-UAV coverage deployment in UAV networks: A game-theoretic framework," China Communications, vol. 15, no. 10, pp. 194-209, Oct. 2018.

[13] J. Sun and C. Masouros, "Drone positioning for user coverage maximization," in Proc. IEEE 29th Annu. Int. Symp. Pers., Indoor, Mobile Radio Commun. (PIMRC), Sep. 2018, pp. 318-322.

[14] J. Lyu, Y. Zeng, and R. Zhang, "UAV-aided offloading for cellular hotspot," IEEE Trans. Wireless Commun., vol. 17, no. 6, pp. 3988-4001, Jun. 2018.

[15] J. Lu, S. Wan, X. Chen, and P. Fan, "Energy-efficient 3D UAV-BS placement versus mobile users' density and circuit power," in Proc. IEEE Globecom Workshops (GC Wkshps), Dec. 2017, pp. 1-6.

[16] M. Mozaffari, W. Saad, M. Bennis, and M. Debbah, "Optimal transport theory for power-efficient deployment of unmanned aerial vehicles," in Proc. IEEE Int. Conf. Commun. (ICC), May 2016, pp. 1-6.

[17] K. Li, W. Ni, X. Wang, R. P. Liu, S. S. Kanhere, and S. Jha, "Energyefficient cooperative relaying for unmanned aerial vehicles," IEEE Trans. Mobile Comput., vol. 15, no. 6, pp. 1377-1386, Jun. 2016.

[18] S. Kandeepan, K. Gomez, L. Reynaud, and T. Rasheed, "Aerialterrestrial communications: Terrestrial cooperation and energy-efficient transmissions to aerial base stations," IEEE Trans. Aerosp. Electron. Syst., vol. 50, no. 4, pp. 2715-2735, Oct. 2014.

[19] Y. Zeng and R. Zhang, "Energy-efficient UAV communication with trajectory optimization," IEEE Trans. Wireless Commun., vol. 16, no. 6, pp. 3747-3760, Jun. 2017.

[20] M. Hua, Y. Wang, Z. Zhang, C. Li, Y. Huang, and L. Yang, "Powerefficient communication in UAV-aided wireless sensor networks," IEEE Commun. Lett., vol. 22, no. 6, pp. 1264-1267, Jun. 2018.

[21] J. Sun, "Energy aware network optimization with aerial base stations ," University College London mphil thesis, 2019.

[22] Y. Zeng, J. Xu, and R. Zhang, "Energy minimization for wireless communication with rotary-wing UAV," IEEE Trans. Wireless Commun., vol. 18, no. 4, pp. 2329-2345, Apr. 2019.
[23] F. Wu, D. Yang, L. Xiao, and L. Cuthbert, "Energy consumption and completion time tradeoff in rotary-wing UAV enabled WPCN," IEEE Access, vol. 7, pp. 79 617-79 635, Jun. 2019.

[24] X. Jing and C. Masouros, "UAV trajectory design and bandwidth allocation for coverage maximization with energy and time constraints," in Proc. IEEE 31st Annu. Int. Symp. Pers., Indoor, Mobile Radio Commun. (PIMRC), Aug. 2020, pp. 1-6.

[25] Q. Shi and M. Hong, "Spectral efficiency optimization for millimeter wave multiuser MIMO systems," IEEE J. Sel. Topics Signal Process., vol. 12, no. 3, pp. 455-468, Jun. 2018.

[26] J. Zhang, Y. Huang, J. Wang, R. Schober, and L. Yang, "Power-efficient beam designs for millimeter wave communication systems," IEEE Trans. Wireless Commun., vol. 19, no. 2, pp. 1265-1279, Feb. 2020.

[27] S. Boyd and L. Vandenberghe, Convex Optimization. Cambridge, U.K.: Cambridge Univ. Press, 2004.

[28] J. Lyu, Y. Zeng, R. Zhang, and T. J. Lim, "Placement optimization of UAV-mounted mobile base stations," IEEE Commun. Lett., vol. 21, no. 3, pp. 604-607, Mar. 2017.

[29] Y. Zeng, R. Zhang, and T. J. Lim, "Throughput maximization for UAVenabled mobile relaying systems," IEEE Trans. Commun., vol. 64, no. 12, pp. 4983-4996, Dec. 2016.

[30] LTE Unmanned Aircraft Systems-Trial Report, Qualcomm, San Diego, CA, USA, Feb. 2017.

[31] N. Li, L. Ting, N. Adila Husna, and H. Husin, "Gps systems literature: Inaccuracy factors and effective solutions," Int. J. Comput. Netw. Commun, vol. 8, no. 2, pp. 123-131, Mar. 2016.

[32] M. Hong, M. Razaviyayn, Z. Luo, and J. Pang, "A unified algorithmic framework for block-structured optimization involving big data: With applications in machine learning and signal processing," IEEE Signal Processing Magazine, vol. 33, no. 1, pp. 57-77, Jan. 2016.

[33] J. Zhang, Y. Zeng, and R. Zhang, "UAV-enabled radio access network: Multi-mode communication and trajectory design," IEEE Trans. Signal Process., vol. 66, no. 20, pp. 5269-5284, Oct. 2018.

[34] Q. Wu, Y. Zeng, and R. Zhang, "Joint trajectory and communication design for multi-UAV enabled wireless networks," IEEE Trans. Wireless Commun., vol. 17, no. 3, pp. 2109-2121, Mar. 2018.

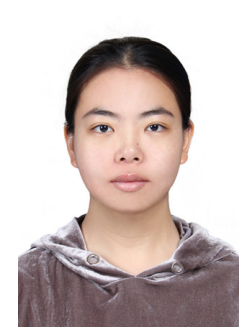

Xiaoye Jing (Student Member, IEEE) received the B.Eng. from Harbin University of Science and Technology, Harbin, China in 2017 and the M.Eng. from Harbin Institute of Technology, Harbin, China in 2019. Now, she is a Ph.D. Student in the Department of Electronics and Electrical Engineering, University College London, UK since 2019. She is currently a Marie Curie Early Stage Researcher supported by the European Union's Horizon 2020 research and innovation programme PAINLESS. Her research interests include UAV communication, joint UAV and radar network and UAV target localization.

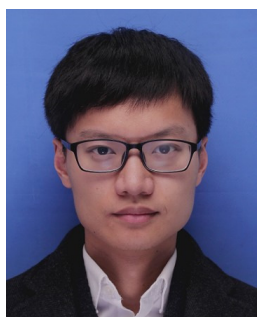

Jingcong Sun received the bachelor's degree (Hons.) from Xi'an Jiaotong-Liverpool University, China, and the University of Liverpool, U.K., in 2016, and the M.Sc. degree (Hons.) from University College London (UCL), U.K., in 2017, where he is currently working in Huawei, Shenzhen, China. His research interests include UAV communications, convex optimization, and spatial modulation. He was a recipient of the UCL's Deans List and Best Overall Student Prize in Telecommunications. 


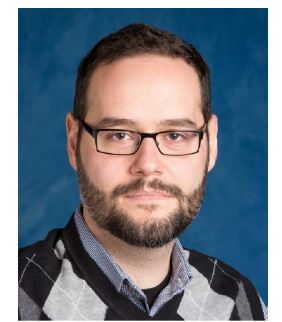

Christos Masouros (Senior Member, IEEE) received the Diploma degree in Electrical and Computer Engineering from the University of Patras, Greece, in 2004, and MSc by research and $\mathrm{PhD}$ in Electrical and Electronic Engineering from the University of Manchester, UK in 2006 and 2009 respectively. In 2008 he was a research intern at Philips Research Labs, UK. Between 2009-2010 he was a Research Associate in the University of Manchester and between 2010-2012 a Research Fellow in Queen's University Belfast. In 2012 he joined University College London as a Lecturer. He has held a Royal Academy of Engineering Research Fellowship between 2011-2016.

$\mathrm{He}$ is currently a Full Professor in the Information and Communication Engineering research group, Dept. Electrical and Electronic Engineering, and affiliated with the Institute for Communications and Connected Systems, University College London. His research interests lie in the field of wireless communications and signal processing with particular focus on Green Communications, Large Scale Antenna Systems, Communications and Radar
Co-existence, interference mitigation techniques for MIMO and multicarrier communications. He was the recipient of the Best Paper Awards in the IEEE GlobeCom 2015 and IEEE WCNC 2019 conferences, and has been recognized as an Exemplary Editor for the IEEE Communications Letters, and as an Exemplary Reviewer for the IEEE Transactions on Communications. He is an Editor for IEEE Transactions on Communications, IEEE Transactions on Wireless Communications, the IEEE Open Journal of Signal Processing, and Editor-at-Large for IEEE Open Journal of the Communications Society. $\mathrm{He}$ has been an Associate Editor for IEEE Communications Letters, and a Guest Editor for IEEE Journal on Selected Topics in Signal Processing issues "Exploiting Interference towards Energy Efficient and Secure Wireless Communications" and "Hybrid Analog / Digital Signal Processing for Hardware-Efficient Large Scale Antenna Arrays". He is a founding member and Vice-Chair of the IEEE Special Interest Group on Integrated sensing and communications (ISAC), and Chair of the IEEE Special Interest Group on Energy Harvesting Communication Networks and an elected member of the EURASIP TAC Signal Processing for Communications. 